\title{
Photonic Crystal Fiber for Sensing Application
}

\section{R. Hemalatha, S. Revathi}

\begin{abstract}
The concern for Sensing Application using photonic crystal had become an elevation in diverse field. Enhanced precision in sensing became an anticipation of users. Photonic crystal fibers are considered to be eccentric than optical fiber sensors due to its geometric structures. By proposing novelty in geometric structures of PCF can increase the sensitivity range as per the required application. In this paper we made a review on different sensors like physical, Curvature, Displacement, Electric and magnetic field, Refractive Index, Bio chemical, Biomedical which are used for sensing applications .

Keywords: Photonic Crystal Fiber, Biochemical PCF Fiber, Biomedical Sensors, Hollow and Dual core.
\end{abstract}

\section{INTRODUCTION}

Philip Russell introduced Photonic Crystal Fiber during the period of 1996. Initially it was defined as solid core. Later during the year of 1998 he introduced hollow core. Photonic crystals are designed with core surrounded by cladding air holes; they are termed as solid core photonic crystal filter. When both core and cladding are structured by air holes they are known as hollow core Photonic crystal fiber [1].
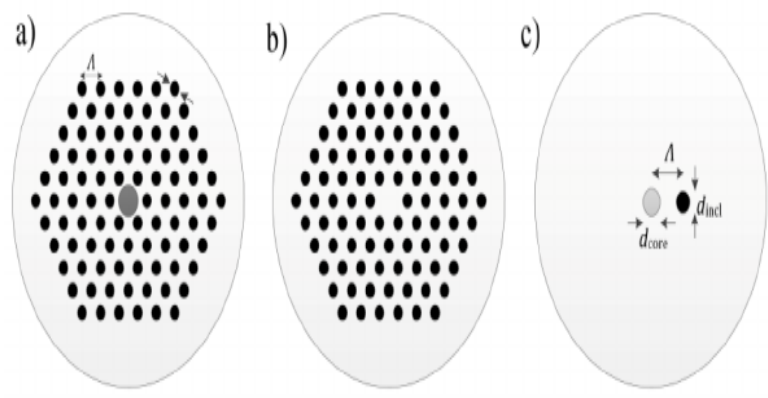

d)

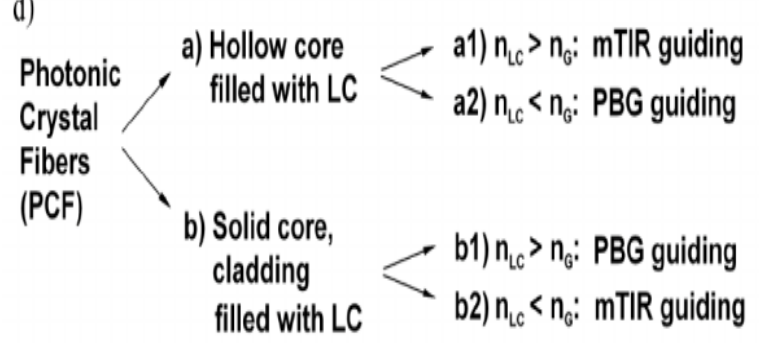

Fig 1. Solid core and hollow core

The above diagram states the basic structure that states hollow core and solid core. These structures can be designed based on the required geometrical structures like oval, spherical, honeycomb, hexagonal, orthogonal, hexagonal and so on.

Revised Manuscript Received on June 05, 2020.

*Correspondence Author

R. Hemalatha*, Research Scholar, School of Electronics Engineering, VIT ,Vellore, India. E-mail: hemalatha.r2019a@vitstudent.ac.in

S Revathi, Ph.D. in Optical Engineering, School of Electronics Engineering, VIT, Vellore, India. E-mail: srevathi@vit.ac.in

(c) The Authors. Published by Blue Eyes Intelligence Engineering and Sciences Publication (BEIESP). This is an open access article under the CC BY-NC-ND license (http://creativecommons.org/licenses/by-nc-nd/4.0/)
The following figure 2, figure 3 , figure 4 shows PCF structures in the form of hexagonal , octagonal, honeycomb structures. PCF follow the properties of photonic crystals which are made by nanostructures. The main advantage of PCF that an optical fiber is light confinement. The Operation is given by their propagation mode. Higher index guiding fibers

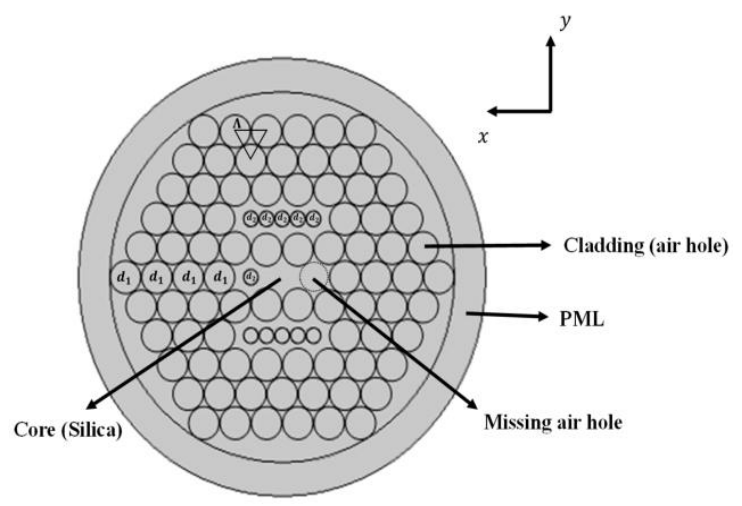

Fig 2. Hexagonal PCF structure

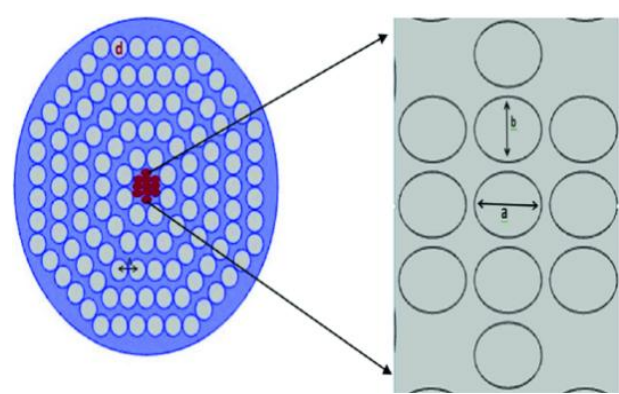

Fig 3. Octagonal PCF structure

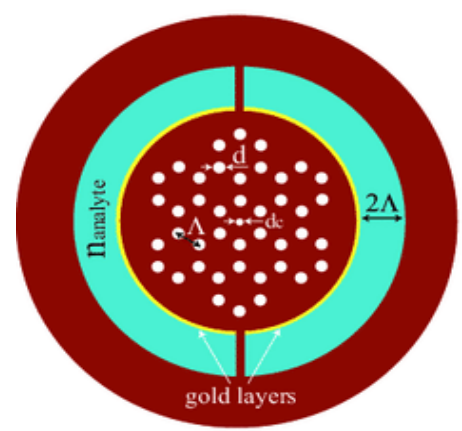

Fig 4. Honeycomb PCF structure

guides in solid core by total Internal Reflection principle. Wherein lower by photonic bandgap effect. There are many categories in photonic crystal fiber. They are, Photonic bandgap fiber which confines light through bandgap effect. Herewith, Holey fiber is also PCF which uses air holes in the cross section. Next type of PCF is hole assisted, which guides through conventional higher core modified. Along with this kind of PCF, Bragg fiber PCF is formed by concentric rings of multilayer film.

Published By:
Plue Eyes Intelligence Engineering \& Sciences Publication (C) Copyright: All rights reserved.

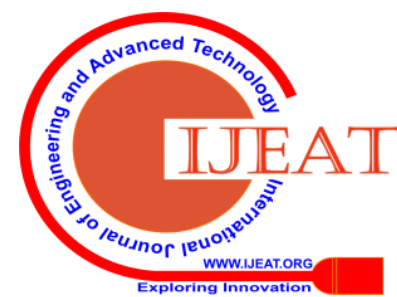




\section{Photonic Crystal Fiber for Sensing Application}

The development of PCF initially started during the year 1997, which proposed single mode PCF with the application of mode filtering, sensors and interferometer. In 1999 PCF with photonic bandgap air core was introduced with waveguide structure. Highly birefringent PCF was introduced during the year 2000 which consists of different air hole diameter with asymmetric core design or two orthogonal axes. Also during this same year Super continuum generation in PCF was generated due to Zero dispersion wavelength and high non-linearity. These were mainly used in laser sound spectroscopy, pulse compression. In 2000 Fabrication of Bragg fiber was introduced for extensive application in fiber lasers and optical sensors. Also double cladding was introduced in the same year with ytterbium doping. During the year 2002 0Ultra-flattened dispersion was introduced. In year 2003 Bragg fiber with air core and silica came into existence. Novel chalcogenide PCF introduced to exhibit an extreme high refractive index coefficient at 2004. Later, kagome lattice PCF was developed in 2005 which has higher contribution to gas sensing. (a) Solid core PCF

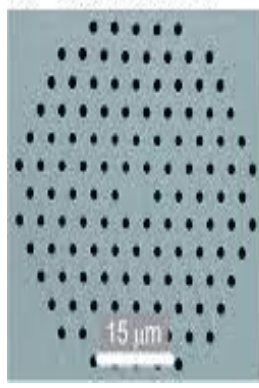

(b) PBG-HCPCF

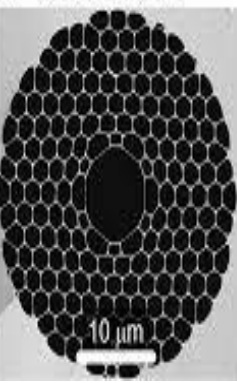

\section{(c) Kagome $\mathrm{HC}$-PCF}

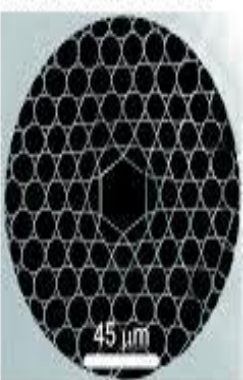

Fig 5. Difference between structures in Solid core PCF, Bandgap effect and Kagome HC-PCF

In 2006, Hybrid PCF was introduced which is doper with germanium. Silicon double Inversion established in 2007 to withstand room temperature. During the year 2009 to 2015 Multicore fiber properties, Nano displacement sensor , equiangular spiral photonic crystal fiber and $\mathrm{Yb}$ doping came into existence. Many other structures were used along with these categories of PCF for better sensitivity [2]. PCF has desired properties for sensitivity or any other application. Dispersion Property which also includes group velocity, material, waveguide and chromatic dispersion. Wherein, loss mechanism is another property which comprises of confinement loss and bending loss. VParameter, Effective area, Numerical Aperture, Spot size, Non- linearity co-efficient is also other properties of PCF. The Fabrication technologies used in PCF are Stack and draw process, Extrusion and filling technology, Sol-gel technique for fabricating irregular shaped PCF. There exits many application on PCF in various field like Sensing Application, Medical Application, Communication Application, Laser Technology, Optical interconnection, Multi structured fiber. In this paper we review on sensing application of Photonic crystal fiber.

\section{SENSING APPLICATIONS}

In-spite of developing sensing application in PCF, Many scientific groups started research on PCF for its unique characteristics. The main attraction towards PCF is its varying locations, size in cladding or core holes. PCF has capacity to propagate light in air. Wherein PCF has also additional capacity to insert gas or liquid into air holes which becomes the main reason to interact with the sample to be sensed. In this, sensing applications of PCF in diverse field are explained. Based on parameter PCF sensing are divided to Physical sensors [3], Biochemical sensors and Biomedical sensors.

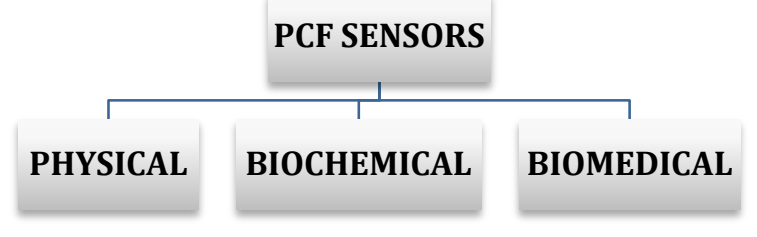

Fig 6. Major types of PCF sensors

\section{A. Physical Sensors}

Physical sensors are beneficial because of its flexibility nature and its internal structure to fill analyte for interaction with light. Physical sensors are mainly meant for measuring some desired parameters like pressure, torsion, refractive index, electric field, Magnetic field, temperature, Displacement, Stress, Strain for various applications in health monitoring, bio-medical sensing, civil structure monitoring, remote sensing, in-order to find the fault in the initial state and to prevent accidents or abnormal conditions.

\section{B. Biochemical sensors}

Some sensors are used to detect chemicals which are in the form of gas, vapour or liquid. They are used chemical industries to sense various chemicals to detect leakages and density of chemicals. In medical field blood components and harmful substance in body are sensed. Food components are also sensed to identify harmful additives.

\section{Biomedical Sensors}

There is more need for sensing in medical field. Blood sensing, DNA hybridisation, to detect disease, to detect antibodies and antigens, to detect pathogens, to detect glucose. Different structures are introduced to increase the sensitivity range. PCF structures are used to sense because of its reliable structures.

\section{DIFFERENT TYPES OF SENSORS}

In this section we will discuss about various types of sensors with its result and its applications.

\section{A. Temperature Sensor}

PCF type are widely used in the fields of biomedicine to monitor medical incubation and dialysis machines. Agricultural field uses to monitor soil and water for plant growth. Food, beverage industries sense meat processing, fermentation process and temperature of storage room is sensed. Birefringence is comparatively low as decrease in the temperature. A methods implements filled ethanol in air holes of PCF. Through this, the varies 45 to $75 \circ \mathrm{C}$ and $L=$ $5.05 \mathrm{~cm}$ [4], which have higher application on detection.

Published By:

Blue Eyes Intelligence Engineering

\& Sciences Publication

(C) Copyright: All rights reserved.

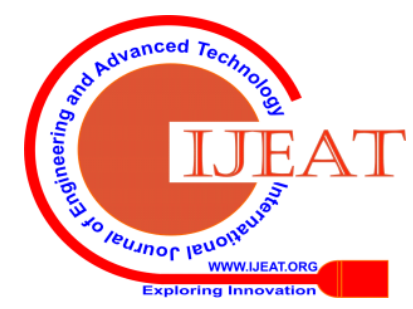


Sensitivity $31.66 \mathrm{pm} /{ }^{\circ} \mathrm{C}$ and displacement range of -528.57 $\mathrm{pm} / \mu \mathrm{m}$, respectively [5].This is achieved through the structure of coated strip. Towards designed hybrid structure senses both temperature and displacement simultaneously. A novel fiber in-line Michelson interferometer been proposed to achieve $-0.05 \mathrm{rad} /{ }^{\circ} \mathrm{C}$ at $20^{\circ} \mathrm{C}$ to $90{ }^{\circ} \mathrm{C}$ [6] inorder to lower measuring error phase- shift, tracking demodulation method used. Sensitivity $14.31 \mathrm{pm} /{ }^{\circ} \mathrm{C}$ using silica cavity [7] at $100-800{ }^{\circ} \mathrm{C}$.
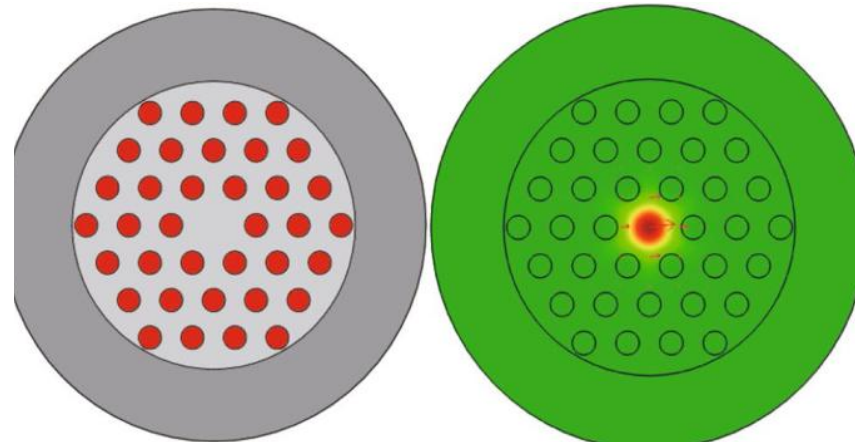

Fig 7. A simple design structure to sense temperature

To detect environmental conditions $0{ }^{\circ} \mathrm{C}$ to $360{ }^{\circ} \mathrm{C}$, two dimensional photonic crystal ring resonator proposed [8]. Silicon rods are in form of Hexagonal rods surrounded by air. Detection range is relied on change in refractive index. $92.3 \mathrm{pm} /{ }^{\circ} \mathrm{C}$. This is suitable for nanotechnology. When PCF is infilterd with liquid crystal, periodical alteration occur due to electro-optic effect as voltage is applied externally. $15^{\circ} \mathrm{C}$ to $58^{\circ} \mathrm{C}$ [9] is the sensitivity range obtained using this sensor. Liquid crystal consists high thermos- optic coefficient used for biochemical application for cell culture. Graphene oxide is coated with PCF and cascaded in two spherical structures. This sensor depends on Mach- Zehnder interferometer. Graphene Oxide is used as it has the property of hydrophilic which changes the refractive index. Temperature sensitivity and humidity sensitivity value found in this research work $0.063 \mathrm{~nm} /{ }^{\circ} \mathrm{C}$ and $0.086 \mathrm{~nm} /{ }^{\circ} \mathrm{C}$ at $10^{\circ} \mathrm{C}$ to $70^{\circ} \mathrm{C}$. Detection are $0.128 \mathrm{~nm} / \% \mathrm{RH}$ and 0.159 $\mathrm{nm} / \% \mathrm{RH}$ from $30 \%$ to $70 \%$ [10]. Novel fluorescein luminescence is introduced to sense wide range of sensing. With the detection limit at the level $0.24 \mathrm{~mW}$ and wavelength presented is 490nm [11]. Dual elliptical core photonic crystal fiber is introduced for wide sensitivity in short range. Thermo -optic co-efficient of silica and chloroform are used to analyse the temperature. Obtained result through proposed method $42.99 \mathrm{~nm} / \mathrm{C}$ for distance $1.41 \mathrm{~cm}$ [12]. Isopropanal filled with photonic crystal fiber long period grating used in high thermos optic coefficient. Detected value $1.356 \mathrm{~nm} /[C$ [13] .

\section{B. Alcohol sensor}

Alcohol may be in the form of liquid or vapour. Its sensitivity are achieved through different structures in Photonic crystal fibers. Octagonal structure is proposed with micro structure porous core to sense aqueous analysts with wavelength range of $0.80^{-1} \mathrm{~m}$ to $2.0^{-1} \mathrm{~m}$. FVFEM is used for numerical analysis. In this work ethanol, methanol, propanol, butanol, and pentanol these solutions are sensed with the sensitivity value of $67.66 \%, 66.78 \%$, $68.34 \%$, $68.72 \%$, and $69.09 \%$ with confinement loss of $2.42 \times 10^{-10}$ $\mathrm{dB} / \mathrm{m}, 3.28 \times 10^{-11} \mathrm{~dB} / \mathrm{m}, 1.21 \times 10^{-6} \mathrm{~dB} / \mathrm{m}, 4.79 \times 10^{-10} \mathrm{~dB} / \mathrm{m}$, and $4.99 \times 10^{-9} \mathrm{~dB} / \mathrm{m}$. Wavelength at $1.33 \mathrm{~m}^{-1}$ [14] .When alcohol is the form of vapour it is sensed through change in colour during light incident. Cellulose film with colloidal array are embedded inside in three dimentional and they are fabricated with poly methacrylate as their refractive index are increased, redshift in reflection of incident light. This changes the color from blue to green visually [15]. Quasifiber sensor is proposed for alcohol sensing. FEM implemented for NA at $0.80 \mu \mathrm{m}$ to $2.8 \mu \mathrm{m}$ wavelength. At the outer boundary scattering boundary condition is implemented to attain sensitivity from $60 \% \sim 98 \%$, Numerical Aperture of 0.68[16], this type of sensor is used in medical imaging. Zeonex based photonic crystal is modeled to identify the ethanol content in food and beverage. The obtained sensitivity $68.87 \%$ and confinement loss at $1 \mathrm{THz}$ are $7.79 \times 10-12 \mathrm{~cm}^{-1}$ [17].

\section{Analyte Sensing}

Analyte is a substance which is in the form of liquid. The substance to be sensed by a photonic crystal fiber is given by an analyte. Octagonal photonic crystal fiber proposed with perfectly matched layer. For sensing wavelength upto $2.2 \mu \mathrm{m}$ starting from $0.6 \mu \mathrm{m}$, The achieved sensitivity for Ethanol, Benzyne andwater, are given by $56.75 \%, 58.86 \%$ and $52.07 \%$ at $\lambda=1.33 \mu \mathrm{m}$. CL $1.55 \times 10^{-13}$ $\mathrm{dB} / \mathrm{m}, 5.05 \times 10^{-13} \mathrm{~dB} / \mathrm{m}$ and $7.50 \times 10^{-13} \mathrm{~dB} / \mathrm{m}$,EA of 4.91 $\mu \mathrm{m}^{2}, 5.11 \mu^{2}$ and $5.31 \mu^{2}$ [18].Another square cored introduced to achieve the sensitivity and $56.25 \%$, $58.36 \%, 51.65 \%$, for above with the confinement loss of $1.31 \times 10-12 \mathrm{~dB} / \mathrm{m}, 3.13 \times 10-13 \mathrm{~dB} / \mathrm{m}, 6.64 \times 10-13 \mathrm{~dB} / \mathrm{m}$ [19].An innovative photonic crystal fiber is introduced with the principle that implements a single mode gets spliced into multimode fiber in either sides. This type of PCF consists of empty core which is surrounded by elliptical plasma rods. E - band is with dispersion of 1360-1460 nm and U-band dispersion of $1360-1460 \mathrm{~nm}$ [20]. In this type of model arrangement different core is for pentanol designed to attain higher sensitivity. Ethanol sensitivity coefficient is given by $32 \%-42 \%$. Water sensitivity co-efficient is givent b1 $8 \%$ 25\% [21]. In this same model the PCF uses three different structures. They are given by X-Model ,Z- Model and VModel.

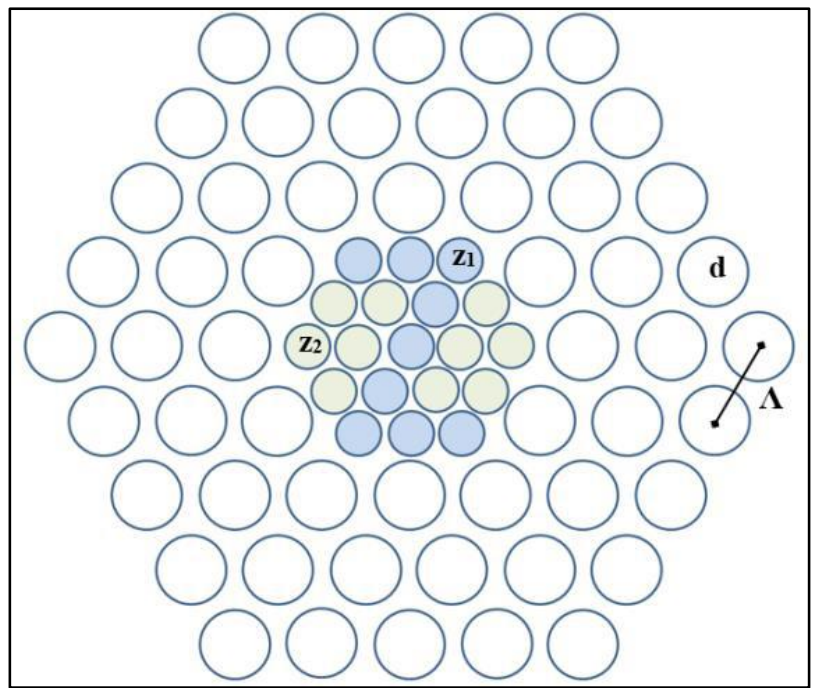

Fig 9. Z-Model PCF

Published By:

Blue Eyes Intelligence Engineering
\& Sciences Publication

(C) Copyright: All rights reserved.

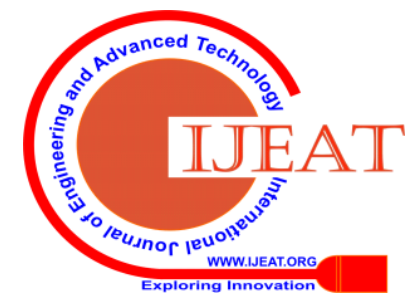




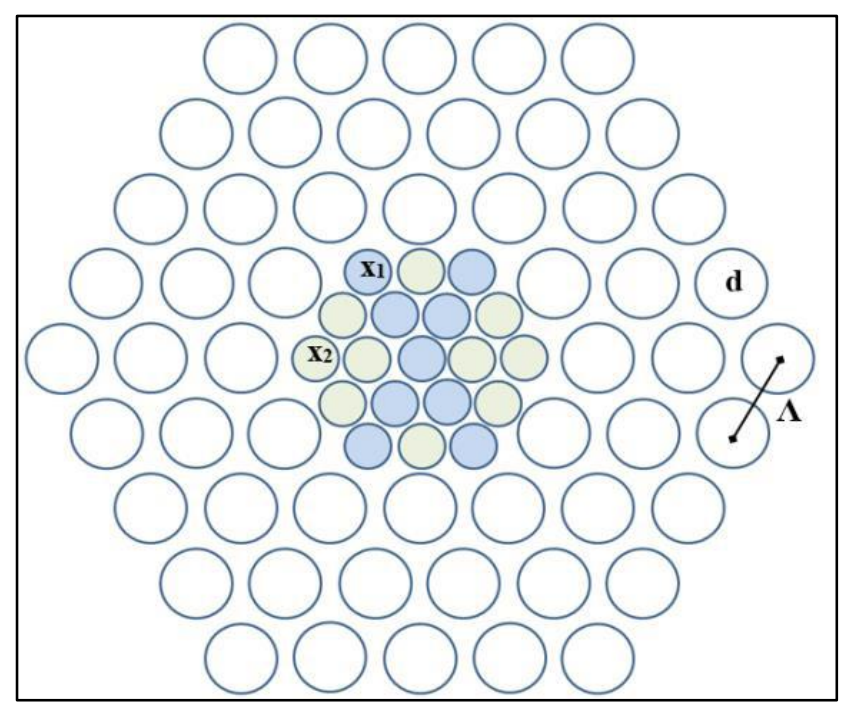

Fig 10. X-Model PCF

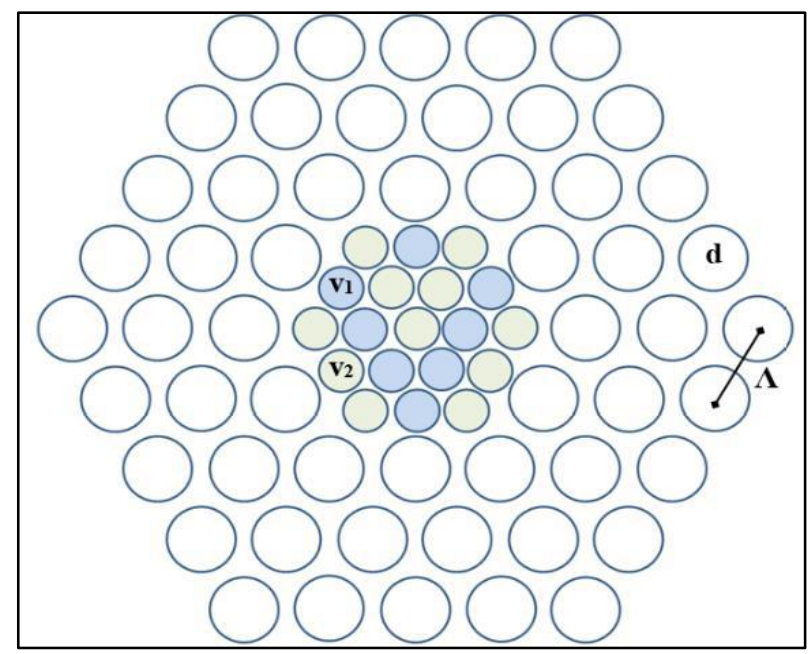

Fig 11. V-Model PCF

\section{Blood Sensing}

Blood is an important component for survival. From normal condition to pandemic condition sensing of blood plays a vital role for finding the problem state. Through sensing of blood detection of glucose, hemoglobin, antigen, antibody, pathogens are also identified. Glucose in salaiva is sensed through haemoglobin directly through Matrix assisted pulsed laser evaporation technique. In this paper haemoglobin in the blood is been immobilized to get the glucose concentration. The obtained glucose concentration is $0.1 \mathrm{mmol} / \mathrm{L}$ [22].In this paper PCF are used with hollow core structure in PCF to sense epidermis blood. The proposed system gives sensitivity of $674.603 R I U^{-1}$ [23] by the model HC19-1550 PCF. There are many components in blood with varied sensitivity. In this paper HC photonic crystal fiber is used to identify the components like water, plasma, WBC, hemoglobin and red blood cells in blood. There is very less confinement loss. Here the frequency used is $2 \mathrm{THz}$.Finally the obtained result through this structure 89.14\%,90.48\%, 91.25\% (WBCs), 92.41\% for hemoglobin (HB), 93.50\% [24]. Same work includes Zeonex as fiber material for high sensititivity. The Fabrication of the material is done through $3 \mathrm{D}$ printing technique. The sensitivity range obtained are given by $55.82 \%$, 58.047\%, $62.721 \%, 65.054 \%$ and $66.469 \%$. Also the obtained confinement losses are given by $(\mathrm{dB} / \mathrm{Km}) 8.1343 \times 10^{-11}$, $4.8787 \times{ }^{10-10}, 1.8134 \times{ }^{10-09}, 2.5928 \times{ }^{10-08}$, and $4.890 \times$ ${ }^{10-08}$ [25].These kind of sensor are used in the form of nanosized biosensor to obtain maximum performance. Dshape elliptical DC Photonic crystal fiber is introduced to obtain sensitivity $4.62 \mathrm{~nm} / \mathrm{gL}^{-1}$ and $5.1 \mathrm{~nm} / \mathrm{gL}^{-1}$ [26]. Following diagram explains above Description. This structure also provides air holes structure

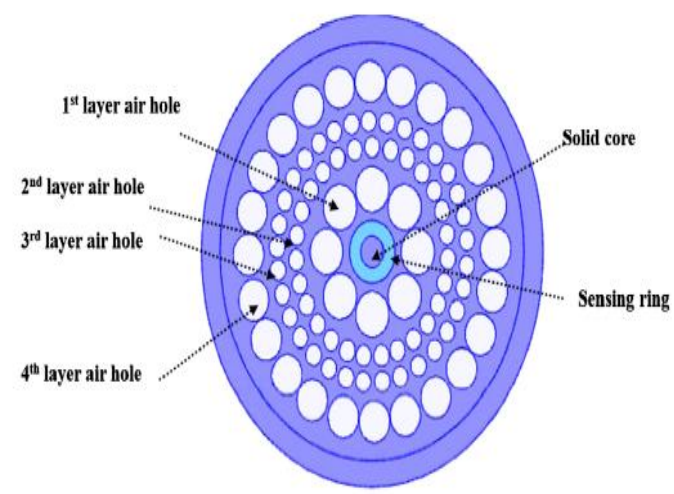

Fig 12. SC-PCF Ring structure

Figure states 5 different layers used for analysing the sensitivity.

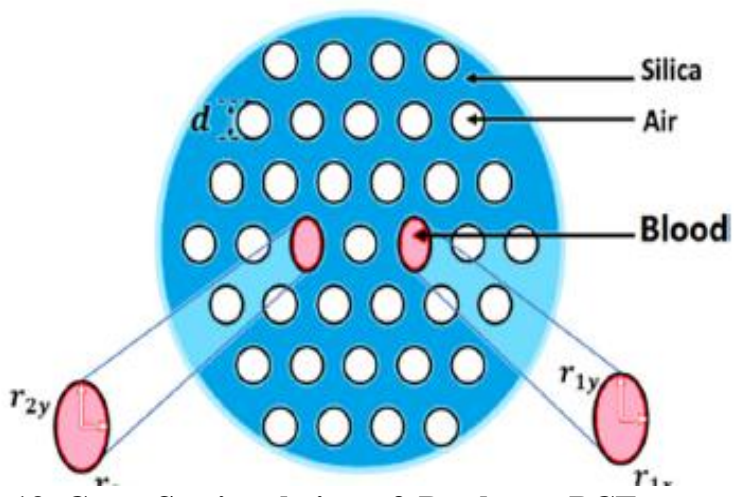

Fig 13. Cross Sectional view of Dual core PCF structure for blood sensing

The above diagram states the structure of Dual core PCF. The diagram clearly explains about presence of blood in air hole.
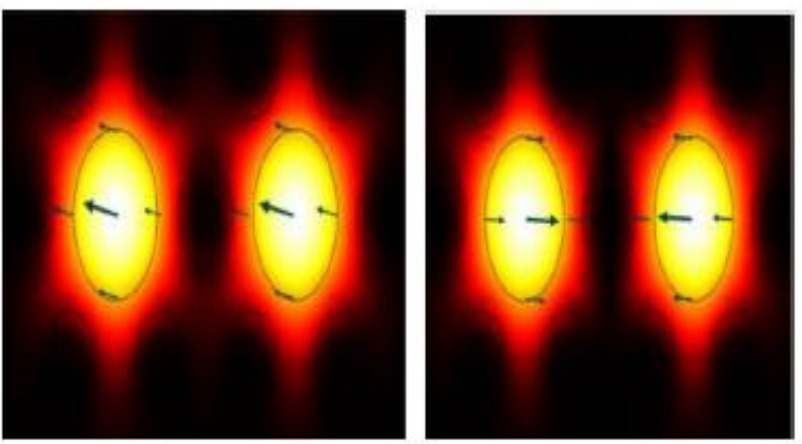

Fig 14. Mode field distribution

Published By:

Blue Eyes Intelligence Engineering

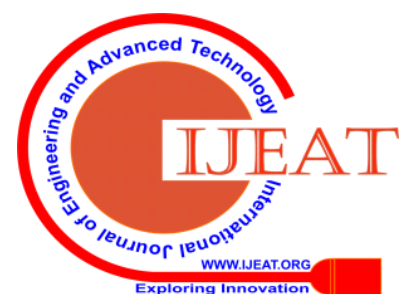


The above diagram explains about the distribution of modes clearly in both polarisations.

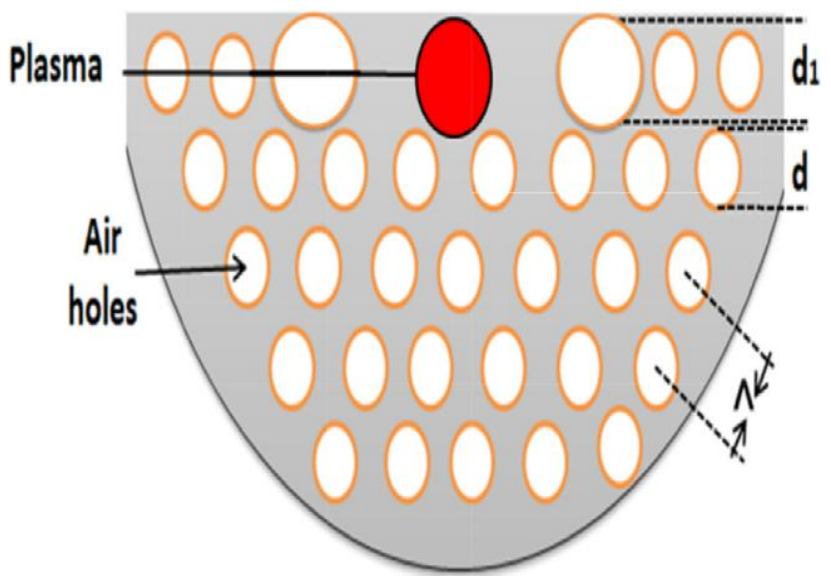

Fig 15. D-shape Structure

These types are used in finding variation in biosamples. Another research paper which explains symmetric photonic crystal fiber for sensing blood which is provided in elliptical core. This structure is based on different polarization and coupling principle. The obtained result through this structure is given by $13.5 \mathrm{~nm} / \mathrm{g} / \mathrm{dL} \mathrm{X}$-polarized mode and 5.96 $\mathrm{nm} / \mathrm{g} / \mathrm{dL}$ Y-polarized mode with $5 \mathrm{~cm}$ fiber [27]. For sensing blood constituents in the wavelength from the range of 1530-1565nm; PC based ring resonator enabled. For Cytop (polymer), Biotin-Streptavidin, Albumin, Ethanol, Bovine Serum Glucosesolution(40gm per 100ml), Hemoglobin, Blood Plasma, Poly-acrylamide [28].Along with Finite Difference Time Domain method, EM Equation Propagation and photonic Bands in simulation tools; used for modelling PCRR signals. Through this design the obtained sensitivity is given by $343 \mathrm{~nm} / \mathrm{RIU}$. This result is achieved by the little change in refractive index [29]. By implementing FVEIM and TMM blood glucose level identified and through D-shaped photonic crystal fiber (DPCF) doped with gold nanoparticles the same parameter can be identified .These are embedded with titanium dioxide which act as an important material for detection 0.83 $\mathrm{nm} /(\mathrm{g} / \mathrm{L})$ and hemoglobin content $160 \mathrm{~g} / \mathrm{L}$ [30]. Another biosensor with dual-core for sensing. Based on Effective RI sample of blood are determined. For the purpose of numerical analysis FV finite element method is used. Attained value are $8.013 \mathrm{~nm} / \mathrm{g} / \mathrm{dL}$ in X-polarized mode and $7.68 \mathrm{~nm} / \mathrm{g} / \mathrm{dL}$ Y-polarized mode under length $3.7 \mathrm{~cm}, 3.2 \mathrm{~cm}$ [31].

\section{E. Chemical sensor}

Chemicals are substance used as mixture in other substance or solvent. It also detects substance and preserve materials We will discuss about PCF based sensors. A proposed D shape is introduced to detect methane, based on Sagnac interferometer. Two ultra-large air holes taken along with methane sensitive film in horizontal way to minimize response time. Methane is in form of gas and its obtained sensitivity is given by $36.64 \mathrm{~nm} \%$ [32] with the range of 0 $3.5 \%$. These type of sensors are mainly used for sensing methane gas. Formalin detection is performed using Kretschmann configuration .In this work graphene and MoS2 are used for detection purpose. The proposed
Published By:

Blue Eyes Intelligence Engineering \& Sciences Publication (C) Copyright: All rights reserved.

biosensor obtained sensitivity is $85.375 \%$ [33]. Detection of bane chemicals are performed in this work. As a fiber material Zeonex is used and performance of sensor analyzed at terahertz mode. Sensitivity obtained is $94.4 \%$ [34] at 1.8 THz frequency with CL $1.71 \times 10-14 \mathrm{~cm}^{-1}$. Sulphuric Acid detection is performed through Comsol software for analysing the sensitivity. At $\lambda=1.5 \mathrm{~mm}$ and confinement loss of $1.422 \times 10^{-17} \mathrm{~dB} / \mathrm{km}$. Finally the attained sensitivity is 63.4\% [35]. photonic crystal fibers with Glass hollow core (HC-PCF) which are modified chemically. Silanol groups are in inner surface of HC-PCF with (3-aminopropyl) triethoxysilane (APTES) [36].

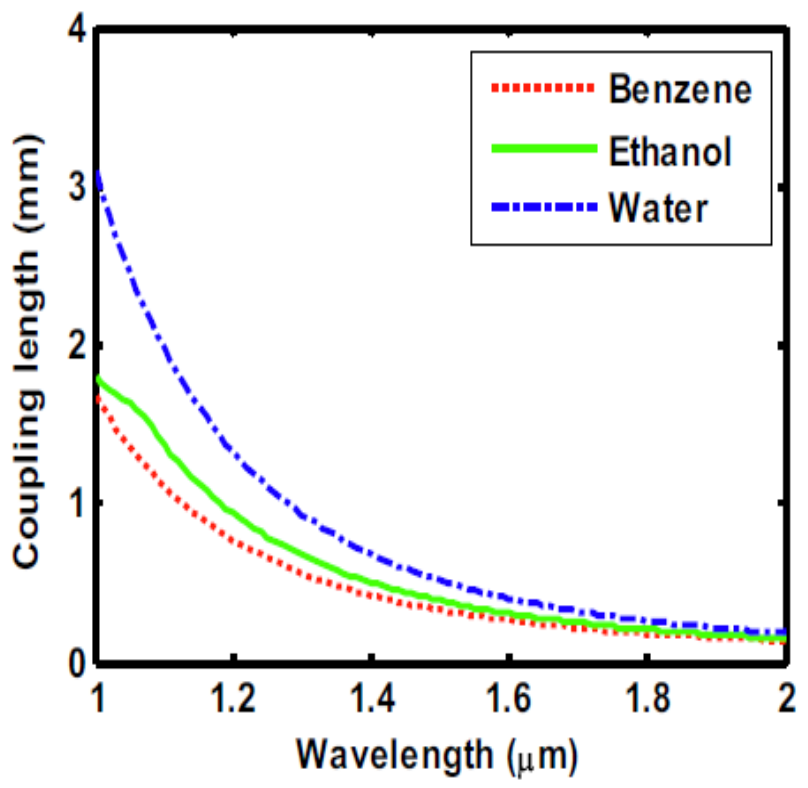

Fig 16. Wavelength measurement

Through the inference from above diagram, there is clear execution in wavelength measurement of Benzene, Ethanol and water.

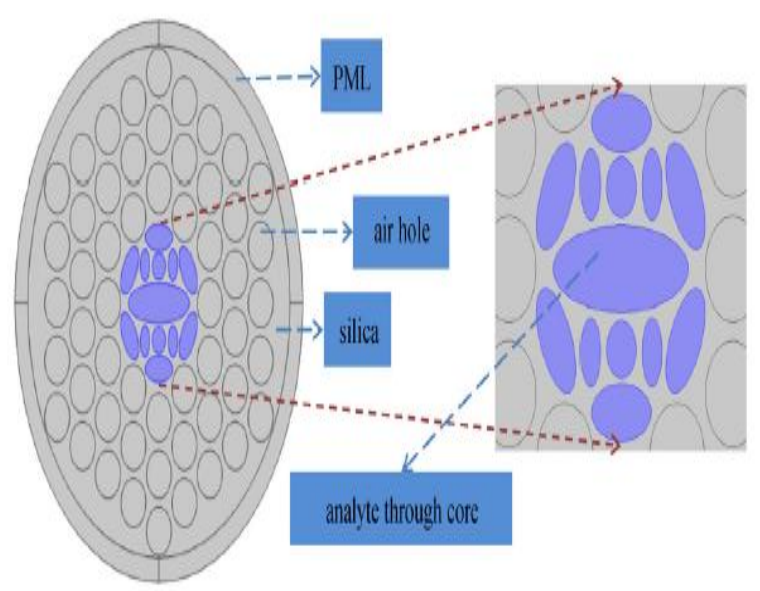

Fig 17. PCF structure used for sensing Sulphuric acid

Microporous core structured PCF chemical sensor introduced which are inbuilt with claddingporous shape . The obtained NA is from NA 0.35 to 0.36 and Aeff from 5.50 to5.66 $\mu^{2}$ [37].

Coblished By:
Blue Eyes Intelligence Engineering
\& Sciences Publication
Copyright: All rights reserved.




\section{Photonic Crystal Fiber for Sensing Application}

In a biochemical sensing Mid-infrared waveband plays a vital role. Silica glass fiber used less because of its high transmission loss nature. Silica glass fiber are used for refractive index testing. For sensing and sampling dualchannel integrated with fluorine-doped tin oxide and fiber sensor proposed for high sensitivity. These structure are error tolerant and useful to detect multiple physical quantities along with biochemical sensing simultaneously [38]. Using COMSOL Multiphysics dual core designed to determine Benzene, ethanol and water are sensed here through PCF separation in its cores.The wavelength used here is $1.55 \mathrm{~mm}$. And the final sensitivity obtained is given by $9615 \mathrm{~nm} / \mathrm{RIU}$ (refractive index unit) [39]. Photonic crystal fiber (RH-CPCF) with hexacore in rotated structure is introduced along with cladding of circular shape in five layers and two layer core are designed. To obtain result PMLB mode of propagation used. Finally at end stimulating sensitivity for Benzene ,Water ,Ethanol are given by $77.16 \%, 73.20 \%, 76.44 \%$, with confinement loss of, $3.07 \times 10-06 \mathrm{~dB} / \mathrm{m}, 2.84 \times 10-02 \mathrm{~dB} / \mathrm{m}, 2.33 \times 10-03$ $\mathrm{dB} / \mathrm{m}$ [40] for wavelength $1 \mathrm{THz}$. Another work which uses spiral photonic crystal fiber along elliptical air-holed placed for liquid sensing. Achieved result 58.3\% 62.7\%,56.8\% of substance propane, propylene, water, with confinement loss of, $7.2 \times{ }^{10-8}, 6.5 \times 10^{-8} \mathrm{~dB} / \mathrm{m}, 8.5 \times 10^{-7}$. Fig 14 explains the structure and analyte used in this work. For the purpose of methane sensing twin-core proposed in which side holes polished with methane sensitive film. This structure is mainly introduced for sensing and monitoring environmental gas. The detection limit is given by 4.60 $\mathrm{nm} / \%$ and $435 \mathrm{ppm}$ [42]. Along with temperature compensation highly sensitive methane sensor is proposed to eliminate cross sensitive effect through matrix demodulation method. Methane sensitivity $20.07 \mathrm{~nm} / \%$ with maximum range value $3.5 \%$ [43]. In this section we saw various structures and results for sensing chemical. Comparing to other sensors chemical sensors requires additional layers of core and cladding to withstand the

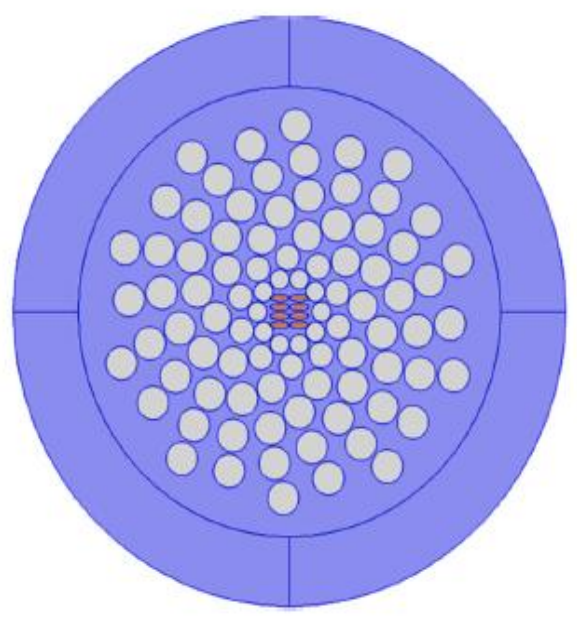

Fig 18. PCF structure with analyte filled

chemical intensitity. Eventhough chemicals are hazardous there is in need of its value for various processing. Hence sensitivity range of chemical and its concentration are essential. The following diagram gives the inference about different layers inside a PCF for sensitivity purpose. This sensitivity is attained through hexagonal air holes.For identification purpose, some of the airholes are structured with separate colour.

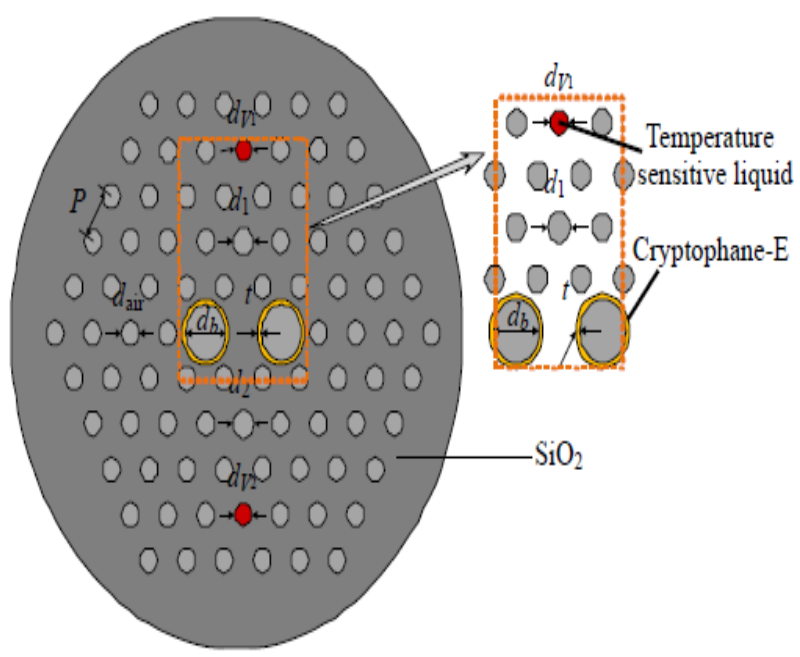

Fig 19. Different layers inside PCF

\section{F. Current Sensing}

Current used in various fields for working and avoiding interruption became important to continue the performance. Such type sensed for proper discharge. CPCF was designed with air holes in circular polarization. Air filling ratio,specific rotation structured by $4.5 \mu \mathrm{m}, 0.42$ and $2.9 \times 10-4 \mathrm{rad} / \mu \mathrm{m}$, [44] respectively. Air hole diameter fluctuation given by $\pm 10 \%$ with higher value to $|S 3|=$ 0.9664 in SSBF. It has circular-polarization that maintains over long period in addition to sensing coil through current measurement as it has decreased error. Also it is low cost with increased accuracy.

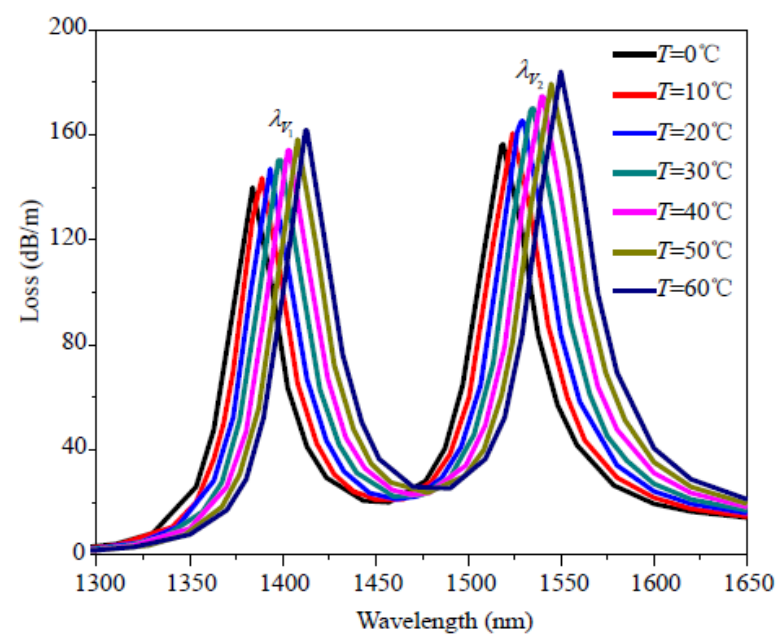

Fig 20. Wavelength Measurement

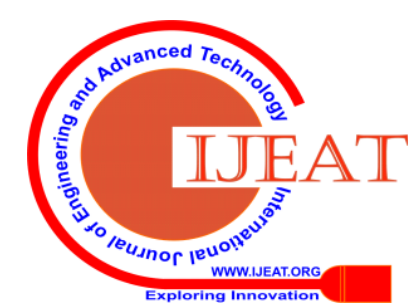




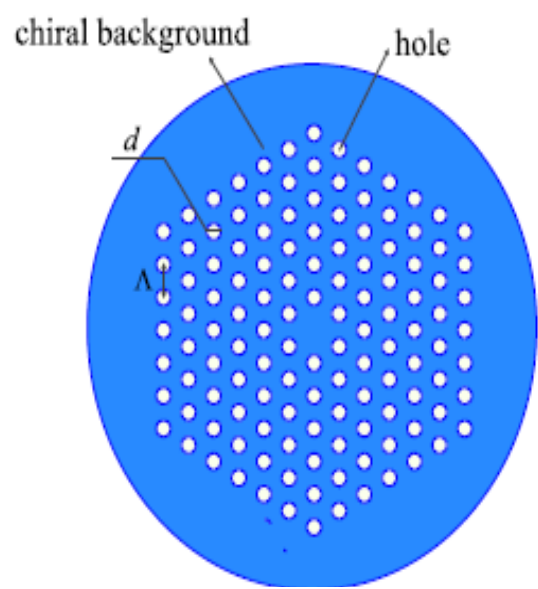

Fig 21. PCF structure with chiral background

\section{G. Curvature Sensing}

In this work, SM fiber taper structure used along loop ring down curvature sensor technique is proposed. Five SMF tapers are used in this work. Curvature is sensed by the ringdown time altered by the bending loss with different curvature. Through concerned work sensitivity is $44.1 \mu \mathrm{m}$ and $65.4 \mu \mathrm{m}$ waist diameters $0.725 \mu \mathrm{s} / \mathrm{m}^{-1}$ and 0.328 $\mu \mathrm{s} / \mathrm{m}^{-1}[45]$.

\section{H. Food Sensing}

Food is an essential for survival, wherein its qualitity is important to stay as safer zone. Some additives are added in food which may become harmful, when level gets exceeded than stipulated range. Hence sensing of food additives plays a vital role. In this paper Hollow Core Photonic Crystal Fiber used to sense Sacchari,Butyl Acetate, Sorbitol,which are harmful food agents. Hexagonal airholes designed at inner cladding for increased sensitivity. Circular airholes are structured. Obtained sensitivity in chemicals are $87.37 \%$, $88.75 \%$ and $86.72 \%$ [46] with operating wavelength 1.33 $\mu \mathrm{m}$. Ethanol also sensed through this structure. Vectorial Finite Element Method used.

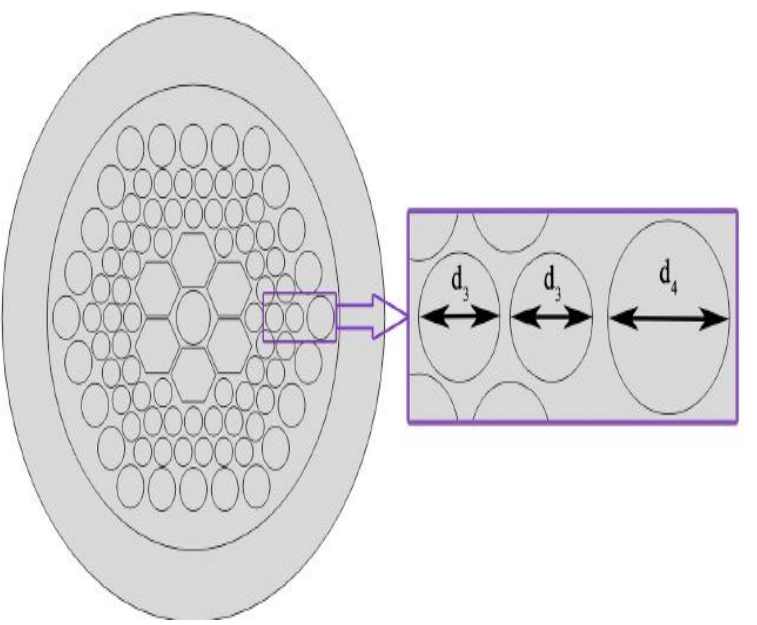

Fig 22. PCF structure hexagonal and circular air holes

\section{Gas Sensing}

Some Gases are colourless and sensing this material is difficult as its spreading is more. In this paper five hexagonal cladding is used with circular shaped core. The obtained sensitivity through this work $56.65 \%$ with CL $2.31 \times 10^{-5} \mathrm{~dB} / \mathrm{m}$, wavelength $1.33^{-1} \mathrm{~m}$, EA $6.44^{-1} \mathrm{~m}^{2}$ [47].
Experiment under Hydrogen Sulphide gas sensed. Graphene structure utilized for better sensing. Air holes in PCF are triangle shape with SOI layer at inner surface. Attained sensitivity through this work is given $1.2 \times 104 \mathrm{~nm} / \mathrm{RIU}$ upto $1.87 \times 10^{-6}$ RIU range in detection [48]. Gas sensitive film introduced for increased sensitivity. Hollow core photonic crystal with group indexed band-gap. For Numerical analysis PWM and FEM utilized. Obtained sensitivity through this work is given by $0.794 \mathrm{~nm} / \%$ [49]. This structure is used for detection of mixed gas also. Spiral shaped Photonic crystal fiber is used here for the detection of toxic and colorless gases. This sensor is mainly used for monitoring air pollution. Two layers of porous core are been micro structured and cladding is structured with spiral shape. NA undergone and sensitive range 55.10\% attained [50]. The absorbed toxic gases through this work are methane and hydrogen fluoride. Dual Fabry-Pérot interferometric carbon monoxide gas sensor introduced. Membrane is coated at one end of optical fiber for increased sensitivity. Single mode fiber is used in another end. These layers are formed by airholes combination. This work is meant for sensing carbon monoxide with the value 0.3473 $\mathrm{dB} \mathrm{m} / \mathrm{ppm}[51], 68 \mathrm{~s}$ and $106 \mathrm{~s}$ are the response and recovery time. This sensor is considered as more advantageous because of its low cost, high sensitivity and compact size. Ambient oxygen, carbon dioxide, nitrogen gases and vapours acetone, 1,1,1-trichloroethane, toluene, are sensed here by implementing hollow core photonic crystal fiber. These types are applied for environmental sensing. Methane and hydrogen fluoride are sensed in this work . The obtained sensitivity and birefringence through the spiral structure are of $57.61 \%$ and $7.53 \times 10^{-3}$ also with $4.1^{\circ}$ divergence and wavelength from $1 \mu \mathrm{m}$ to $1.8 \mu \mathrm{m}$ [52]. The following structures describes the coating and layers in proposed PCF.

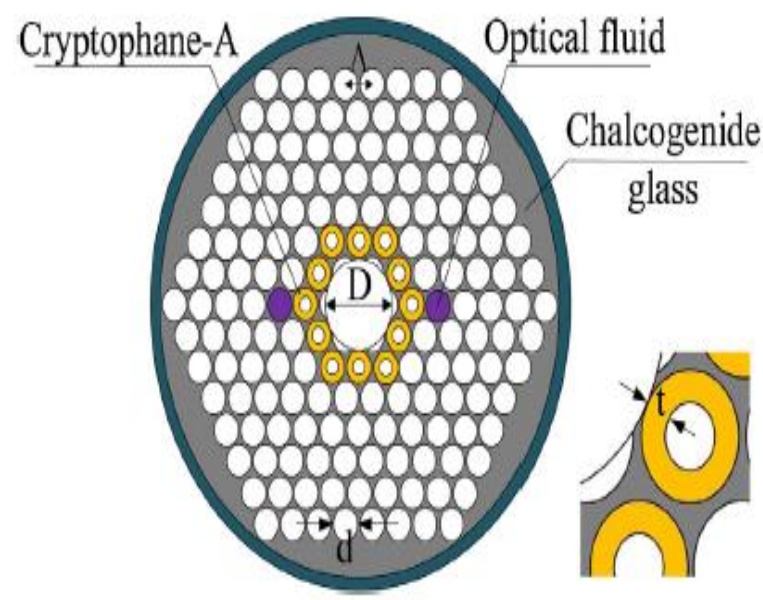

Fig 23. PCF structure explaining its layers

Another research work which works on photon energies that corresponds polar materials that reaches terahertz regime to sense toxic gases. Rotational transitions create weak dipole moment that limits gas at lower concentration.

Published By:

Blue Eyes Intelligence Engineering

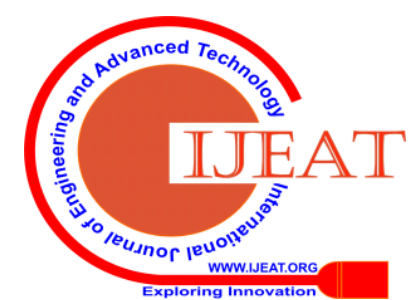


High sensitive detection of gas are achieved through cavity defect stage along resonance on absorption of required characteristic. Hydrogen cyanide of 2 ppm concentration Under 1 atm AP [53]. Circular based gas sensor are analysed. Spiral porous Core region is suggested for high light interaction. Achieved sensitivity are represented as $72.04 \%$ with the wavelength of $1.33 \mu \mathrm{m}$ [54]. In this work toxic gases can be sensed between the wavelength of $1 \mu \mathrm{m}$ to $1.8 \mu \mathrm{m}$. Similar to chemical sensing gas sensing are also challenging as its characteristics are like highly toxic capacity, colourless, odourless. Gases becomes an omnipresent hence we need to identify its intensity and the type of gas. Gas sensors are mainly in used in industries and for environmental purpose to monitor air pollution. To avoid accidents of gas spilling in industries sensors are essential. Hence different structures are introduced to attain better sensitivity.

\section{J. H. Glucose sensing}

Glucose is present in blood, water and many other substances. Glucose is essential but usage in correct density is required. In this section there is a discussion about glucose sensing. Using COMSOL multi physics software silver nanowires are designed with photonic crystal fiber. The attained sensitivity through this work is $19009.17 \mathrm{~nm} / \mathrm{RIU}$ and detected amount of glucose in water is $44.25 \mathrm{mg} / \mathrm{dL}$ [55]. The diameter used here $90-120 \mathrm{~nm}$. In this new work $146699.26 \mathrm{~nm} / \mathrm{RIU}$ [56] is obtained through its refractive index. Urea, Albumin in glucose are analysed using the PCF structure implemented with glucose sensor.This has tighter confinement. Another PCF design got higher refractive index and spectral sensitivity of $19135.70 \mathrm{~nm} / \mathrm{RIU}$ [57]. Air hole designed in such a way that it supports liquid core. In the second layer six cores are present to exit the air hole. This structure is mainly used in real time application. Even though sensors are available users always expect a compact size. In this work the design explains about A compact glucose sensor is designed. For the same PCF is fiber inserted in Sagnac loop interferometer . Spectral sensitivity attained are given by of 22130 $\mathrm{nm} / \mathrm{RIU}$ with the range $0.76 \mathrm{mg} / \mathrm{dL}$ of glucose in water, which is lower than $70 \mathrm{mg} / \mathrm{dL}$ [58]. It is highly efficient for glucose detection in hypoglycemia patients. New glucose sensor in a tapered structure is introduced that are coated with graphene oxide and gold nanoparticles. The taper region is used for exposing evanescent waves at the cladding boundary with graphene oxide . Gold Nano particles and Graphene Oxide are used for its good absorbance capability. Its absorbance wavelengths are given by $230 \mathrm{~nm}$ and 519 nm. The analysis are done through UV-vis spectrophotometer and TEM. The attained value through this structure are $1.06 \mathrm{~nm} / \mathrm{mM}$ [59]. Blood samples of humans are sensed. Coupling is done between silica mode and analyte mode and reported sensitivity is $8333 \mathrm{~nm} /[R I U]$ in variation $10 \mathrm{~g} / \mathrm{l}$ to $20 \mathrm{~g} / \mathrm{l}$ with resonance wavelength [60].Thus we saw different results and structures in glucose sensing among various field including blood samples. Additives in glucose that is toxic in characteristics are added. To overcome this issue, obtaining higher sensitivity is essential and hence different stuctures are implemented.

\section{K. Humidity sensing}

Humidity necessary to sustain suitable condition. In this section exhibits some sensing structures on humidity. PCF coated with car-boxy methyl cellulose for responsive parameter. Bragg grating used for actuating mechanical strain in response to changes. Achieved range is $5 \%-40 \%$ and measurement uncertainty over this relative humidity range is $\pm 2 \%(\mathrm{k}=1)$ [61].Interferometric sensor are spliced into standard single mode fibers, poly(allylamine hydrochloride) and (acrylic acid) ,polymeric nanocoating are deposited on well-established layer-by-layer nano assembly technique. Nano layer modified by humidity as detectable shift is observed in interference pattern. For spectrum analysis Fast Fourier Transform are used response. The resolved humidity through this work is given by $0.074 \%$ and relative humidity is operated at 20-95\% $\mathrm{RH}$ [62]. Finally the response time is obtained as 0.3 . Through a different new inline mode Mach-Zehnder interferometer with coating of graphene quantum dots and polyvinyl alcohol for sensing. The sensitivity gained through this work is $343.7 \mathrm{~nm} / \mathrm{RIU}$ [63].One dimentional photonic crystal fiber is calculated both theoretically and experimentally to detect environmental sensitivity. Multilayer porous thin films are deposited on fiber which are the sensing elements. Humidity detection level is from 11 to $84 \%$ RH [64].

\section{Liquid Sensing}

Liquids are of many forms. Under this section, there is a discussion about different liquid sensors. To detect liquid analytes. Photonic Crystal Fiber with Finite Element Method is used For wavelength $(0.6 \mu \mathrm{m}-1.6 \mu \mathrm{m})$ [65]. It has lower confinement loss. Wherein with the structure of $\mathrm{A}$ solid core for identifying chemicals in liquid form . The wavelength range between 1.4 and $1.65 \mu \mathrm{m}$ are synthesized. The filled amount are glycerol $(n=1.4722)$, ethanol $(\mathrm{n}=$ 1.354), and toluene ( $\mathrm{n}=1.4968)$, By applying various parameters in structures of PCF $65.16 \%, 61.65 \%$, and $64.05 \%$ [66] of sensitivity are attained as a result. Comparatively Confinement loss is minimum through this structures also effective area is analysed here.

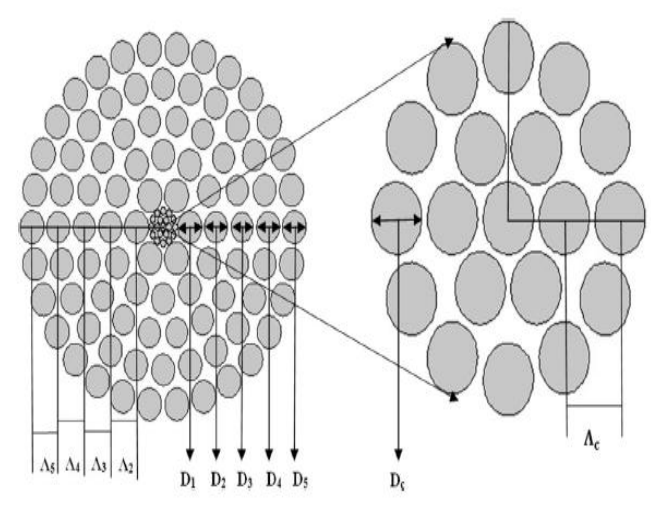

Fig 24. PCF structure with increased number of core and cladding air holes for increased sensitivity

Published By: DOI: 10.35940/ijeat.E9613.069520

Journal Website: www.ijeat.org
Blue Eyes Intelligence Engineering
\& Sciences Publication (C) Copyright: All rights reserved.

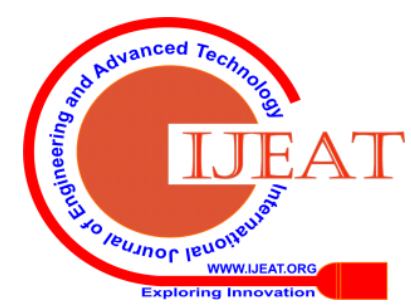


For attaining high sensitivity,high birefringence and low confinement loss in liquid sensinge through Photonic Crystal Fiber, full Finite Element Method for elliptical holes are choosed in the core region. The wavelengths $(1.3 \mu \mathrm{m}-2$ $\mu \mathrm{m})$ are assigned here. 49.13\% [67] is the sensitivity obtained and the birefringence is 0.008. These are suggested for bio-sensing applications. Mach-Zehnder modal for sensing concentration of $\mathrm{Fe}$ in water. The core and cladding are recombined. The sensitivity detected is sensitivity was $42.446 \mathrm{~nm} / \mathrm{RIU}$ [68].

\section{Magnetic Field Sensing}

Magnetic have capacity to attract metallic substance. For sensing magnetic materials these sensors Multimode-single mode-multimode combination is introduced in Magnetic fluid-filled optical fiber Mach-Zehnder interferometer, fabricated through splicing Magnetic fluid-filled capillary tube is sealed within a microstructure. The change in refractive index is due to variation in Magnetic field. Through this $0.12306 \mathrm{~nm} / \mathrm{mT}$ [69] value is attained. By tuning the structure highest response is $5000 \mathrm{pm} / \mathrm{Oe}$ and resolution 11.33 Oe [70] .These type of sensors are mainly used in nanofluidic technology. Magnetic field are also based ferrosoferric oxide to increase sensitivity. There is need to detect magnetic field from 100 Oe to 160 Oe. Attained sensitivity in $\mathrm{X}$ and $\mathrm{Y}$ polarization given by $83268.46 \mathrm{~nm} / \mathrm{RIU}$ and $83188.25 \mathrm{~nm} / \mathrm{RIU}$ [71]. The accuracy is comparatively high with lost cost. 2D photoniccrystal is used with nanocavity infiltrated along with magnetic fluid. To increase the sensitivity air holes which are infiltered are also used in huge number. Around 12 infiltrated airholes are implemented in this structure. Refractive index sensitivity for the structure is provided by $146.97 \mathrm{~nm} /$ refractive index unit [72]. Another D- shaped photonic crystal fiber and Ethanol inserted structure is introduced. Along with magnetic field, temperature also sensed through this work. Magnetic sensitivity is obtained by this structure is $0.21 \mathrm{~nm} / \mathrm{Oe}$ and temperature -1.25 $\mathrm{nm} /{ }^{\circ} \mathrm{C}$ [73]. Square lattice dual core photonic crystal fiber is implemented to attain the sensitivity of sensitivity of 799.07 $\mathrm{pm} / \mathrm{Oe}$ [74]. The structure consists of two cores in which mode coupling is performed. This sensor is used in health monitoring instruments and it is a portable device. Sagnac interferometer based polarization maintaining photonic crystal fiber is used which is a refractive index sensitive structure. Possessing low refractive index, the water-based nanoparticles uses $\mathrm{Fe} 3 \mathrm{O} 4$. In this structure magnetic field it attained in opposite direction. Result obtained through this work is R-squares are $0.98113,0.98415$ respectively for detected range 410-600 Oe [75].

\section{N. Fuel Sensing}

Fuel is essential thing for performing some works like transports and working in generators. If there is adulteration in fuel then it may lead to other functional problem in parameter used. Hence sensing fuel plays vital role to identify adulteration. In this work dual core photonic crystal fiber which consists of single analyte channel is given for detection of petrol adulteration. Using finite element method ,sensing Probes analyzed. Petrol is sensed in circular analyte which is infiltered. Through simulation
20,161.2 nm/RIU is observed [76]. With the help of this work along with the Fuel

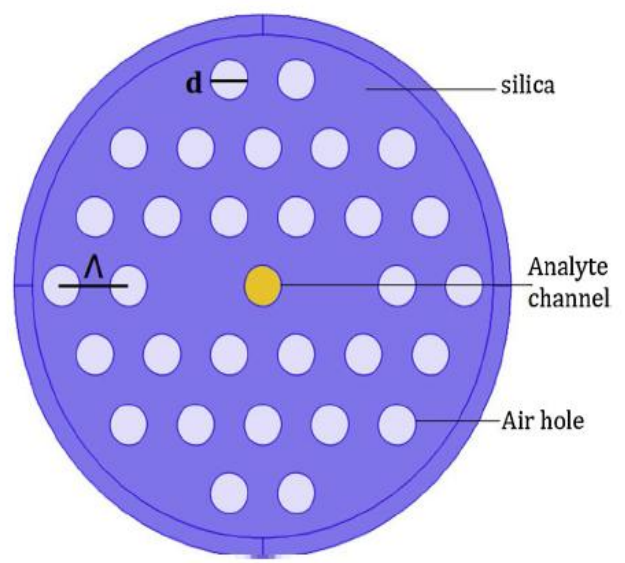

Fig 25. PCF structure used for fuel sensing

adulteration sensing, It can also be used for sensing environmental pollution, to increase the life time of engine.

\section{O. Pressure}

These have physical quantity for observing environmental condition at required application areas, monitoring industrial processes in harsh climate. Interact with human and other animal medical diagnosis purpose, measure human body fluid pressure, under water pressure. Along with this temperature can also be sensed. Pressure measurements are needed for industrial applications at tough environments like power plants, , oil ,gas exploitations are complex in some applications as high temperature and corrosive materials or interference due to electromagnetics, these are overcome my pressure sensors. Measurements of hydrostatic pressure are also done through this sensor. In this section, there is a discussion about its applications and different structures and materials used to increase sensitivity of pressure. modulation and polarisation type used to execute highbirefringence fiber.. Sensitivity obtained through this work is is $2.05 \times 10^{-6}$ [77] RIU/MPa in pressure 0-100 MPa, and temperature sensitivity is $7.27 \times 10-10 \mathrm{RIU} /{ }^{\circ} \mathrm{C}$ upto $0-100$ ${ }^{\circ} \mathrm{C}$. Results show that $0.145 \mathrm{~dB} / \mathrm{MPa}$ at $0-44 \mathrm{MPa}$ of pressure value.These sensors are used in Ocean bottom holes. Polarization is maintained by using SM fiber. 2 Orthogonal modes used inthe PM-PCF are excited by splice point. Wavelength-pressure coefficient $4.15 \mathrm{~nm} / \mathrm{MPa}$, temperature coefficient $1.4 \mathrm{pm} /{ }^{\circ} \mathrm{C}$ [78] . Another novel asymmetric double-core fiber which provides hydraulic pressure is introduced with $10-\mathrm{cm}$ and $2.867-\mathrm{cm}$ of sensing range from 0 to $900 \mathrm{MPa}$ and sensitivity $0.3 \mathrm{~mm} / \mathrm{MPa}$ [79]. Double fiber core with and two side air holes are used for ensuring asymmetric pressure-induced phase change for guiding light in two fiber cores. Using novel hybrid-dual core photonic crystal fiber at a length $6 \mathrm{~cm}$ has sensing range from 0 to1000 Mpa and sensitivity $-11.6 \mathrm{pm} / \mathrm{Mpa}$ [80]. Two-dimensional hexagonal photonic crystal ring resonator structure designed for sensing both pressure and temperature based on effective RI .This nano type sensor is designed to detect pressure upto $6 \mathrm{GPa}$ and temperature 540 ${ }^{\circ} \mathrm{C}$ maximum [81] .

Published By:

Blue Eyes Intelligence Engineering

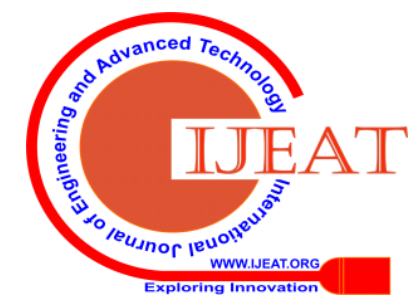




\section{Photonic Crystal Fiber for Sensing Application}

In this work finite-difference time domain method used for functional analysis of dynamic range, sensitivity, transmission efficiency, and quality factor. This sensor is used for bio sensing applications.It is advantageous because of its compact size.

\section{P. Refractive Index}

Based on Refractive index value the other sensors are diagnosed for its sensitivity. With variation in RI shows detecting range in sensor. Through this section,A multi-core micro nano fiber proposed for lower valued solutions. The mode field distribution are tapered multi-core fiber. At length of $16.20 \mathrm{~mm}, 5815.50 \mathrm{~nm} / \mathrm{RIU}$ [82] is obtained. Measurement error is $0.5 \%$. Diameter is less than $4.864 \mu \mathrm{m}$ with sensing rangec $10000 \mathrm{~nm} / \mathrm{RIU}$. This kind of sensors are useful for detecting temperature also it can be used for sensing salinity in seawater. D-shaped with gold belt introduced. There is broad measurement range under 1. 9 $\times 10^{-5}$ RIU [83].With simple structure Mach-Zehnder interferometer demonstrated through fabrication and welding of a dual-waist photonic crystal fiber. Fusion technology and taper in core are performed here. The measured sensitivity through this work is of $263.5 \mathrm{~nm} / \mathrm{RIU}$ [84].The impregnation on anodic aluminum oxide films is introduced to sense water-ethanol and water-glycerol of $\Delta \lambda / \Delta \mathrm{n}=142 \mathrm{~nm} / \mathrm{RIU}$ [85] . Liquid mixtures are identified in this work. Through infiltration method different kinds of liquid mixtures are sensed. By presenting a carbon nanotubes deposited photonic crystal fiber along MachZehnder interferometer configuration RI sensing. CNTs deposited on PCF surface for enhancing interaction of evanescent waves. Sensitivity $19.4 \mathrm{~dB} / \mathrm{RIU}$ within range 1.33 to 1.38 and $24.2 \mathrm{~dB} / \mathrm{RIU}$ under 1.38 to 1.42 [86]. Conventional graded-index multimode optical fiber uses. $\mathrm{NaCl}$ solution to get $70 \mathrm{~nm} / \mathrm{RIU}$ at 1.333 to 1.345 [87] range.Better contact with surface and sensing element. Chemicals and biological sensing can be sensed through this work 2D photonic crystal worked with air-slot widthmodulated line-defect microcavity with waveguide. Though simulation sensitivity of $400 \mathrm{~nm} / \mathrm{RIU}$ [88] with detection limit $2.98 \times 10^{-5}$ RIU is attained. Another D-shaped nearinfrared spectrum suitable for detection of biomedical and biochemical analytes with different indexes. The active metal used here is Gold and a layer of molybdenum disulphide is coated for oxidation resident. Along with this graphene also deposited. Through this simulation simulation the sensitivity attained 14,933.34 nm/RIU with figure of merit, effective RI resolution of 401.05 RIU-1 and $6.69 \times$ 10-6 RIU [89].Similar D-Shape structure with silica as coating material provides wavelength sensitivity 66666.67 $\mathrm{nm} / \mathrm{RIU}, \mathrm{AS}=-1488.82 \mathrm{RIU}-1$, of $\mathrm{NA}=1.36$ to 1.39 , Resolution $=9.66 \times 10-4$ RIU [90]. This sensors are proposed for the analyte sensing in biochemical and biomedical applications. Mach-Zehnder interferometer with Cladding etched for high sensitivity is proposed along with splicing for increased refractive index. MZI with $35 \mathrm{~mm}$ PCF are $211.53 \mathrm{~nm} / \mathrm{RIU}$ and $359.37 \mathrm{~nm} / \mathrm{RIU}$ [91]. These biosensor is used for sensing higher spectral density. Amplitude sensitivity 11,412 RIU-1, when refractive index varied from 1.44 to 1.45 [92]. Coupling is easier through this design. The simulation concludes analyte RI is 1.425
1.45 and 1.45-1.46, with average sensitivity 60,611 and $28,000 \mathrm{~nm} / \mathrm{RIU}$ respectively, and detection limit $1.58 \times 10-6$ and $2.86 \times 10-6$ RIU [93]. An elliptical cavity for detection unit in a $2 \mathrm{D}$ hexagonal hole-type photonic crystal is notable for large detection range $n=1$ to over 2 [94]. Sensing is divided into two distinct detection space in a different spectra. The design structures are analysed through the topology by FDTD and PWE methods. D-shaped infrared spectrum is proposed for analytes and liquids detection with different refractive indices ranging upto 1.40.Gold and graphene are coated to increase the sensitivity $33,500 \mathrm{~nm}$ RIU-1 with resolution $2.98 \times 10-5$ RIU [95]. Hollow core fiber is cost effective, analysed through full vectorial finite element method .This design exhibits sensitivities 10560 $\mathrm{nm} / \mathrm{RIU}, 6400 \mathrm{~nm} / \mathrm{RIU}$, $12,400 \mathrm{~nm} / \mathrm{RIU}$ for, gold silver,copper with resolution of $1.61 \times 0-6$ RIU [96].

\section{Q. Salinity Sensing}

Salinity is increased amount of salt content. Under this section its sensing provided. Seawater said to be highly saline. Sensing is difficult because sensors used corrode due to its high salt content and long-time survival possibility less because of high salt water content. Providing supply for working of sensor also difficult. Cavity photonic crystal resonator proposed for sea water salinity concentration detection . Computed value $544 \mathrm{~nm} / \mathrm{RIU}$ with quality factor 3860. figure of merit $1.234 \times 103$ RIU-1 and detection limit $8.1 \times 10-5$ RIU [97]. Transmission characteristics of device attained through finite-difference time-domain. Result is $37,500 \mathrm{~nm} / \mathrm{RIU}$ and $7.5 \mathrm{~nm} / \%$. Maximum resolutions $66 \times$ $10-06$ RIU and $1.33 \times 10-02 \%$ with linearity 0.9924 at 2.20 cm length [98].This result is obtained through Sagnac Interferometer based fiber. When saline water infiltrated in one of cladding air hole, that offers high confinement loss. In this work along silica Phase matching condition used for power transmission in core. Numerical calculation is done through Finite Element Method. The observed result is given as5405 nm/RIU -X-polarization and $5675 \mathrm{~nm} / \mathrm{RIU}$ ypolarization over 0.0037 RIU limit [99]. This gives $10^{-3}$ birefringence

\section{R. Strain Sensor}

Some of the strain sensor based three interferometric kind fiber explained. Hi-Bi PCF ,PMF and standard used. This type achieves sensitivity $0.893 \mathrm{pm} / \mu \varepsilon, 26.85 \mathrm{pm} / \mu \varepsilon, 1.809$ $\mathrm{pm} / \mu \varepsilon$. for $7 \mathrm{~cm}$ and $23.9 \mathrm{~cm}$ [100]. They are used for measuring hazardous environments and monitoring heath under complex structures. Under cascaded multimode fiber fusion spliced single mode fiber in either ends.. Through this work observed value $\sim-14.89 \mathrm{pm} / \mu \varepsilon$ [101]. Ultra- long period fiber grating sensor introduced for simultaneous measurement. Fabricated done by splicing multimode fiber. Through strong refractive index, uncertainty values of the SMS-ULPFG structure are $3.26 \mu \varepsilon$ and $0.23{ }^{\circ} \mathrm{C}$ over the ranges of $0-1000 \mu \varepsilon$ and $26-118{ }^{\circ} \mathrm{C}$.[102]. Through study of polarization modulation, high-birefringence fiber selected for fluid medium pressure measurement.

Blue Eyes Intelligence Engineering

Retrieval Number: E9613069520/2020@BEIESP

DOI: 10.35940/ijeat.E9613.069520

Journal Website: www.ijeat.org

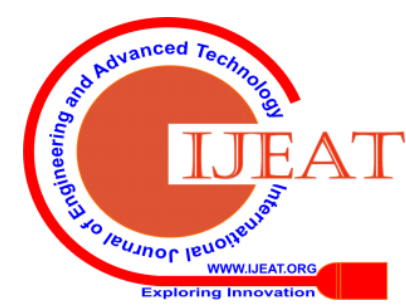


Results show pressure $2.05 \times 10-6 \mathrm{RIU} / \mathrm{MPa}$ in the pressure range of $0-100 \mathrm{MPa}$, and temperature $7.27 \times 10-$ $10 \mathrm{RIU} /{ }^{\circ} \mathrm{C}$ at range of $0-100{ }^{\circ} \mathrm{C}$. Results project pressure sensitivity 0.145 dB/MPa under 0-44 MPa [103] Spliced twin-core fiber with fiber tapers are weak and T-shaped. Roles are splitting and combining modes. Proposed extinction ratio of $20 \mathrm{~dB}$ is achieved. When stress to 841.5 $\mu \varepsilon$, wavelength of $3.31 \mathrm{pm} / \mu \varepsilon$ and $6.11 \mathrm{pm} / \mu \varepsilon$ are achieved. Intensity sensitivities of $7.5 \times 10-3 \mathrm{~dB} / \mu \varepsilon$ and $9.9 \times 10-3$ $\mathrm{dB} / \mu \varepsilon$ [104]. These sensors are used for Practical applications. For a Mach-Zehnder interferometric ,Designed with hollow-core photonic bandgap fiber to detect intrusion. result shows NR in HC-PBG is about $3.6-5.3 \mathrm{~dB}$ increased than SMF upto frequency range $2 \mathrm{kHz}$. Measurements on HC-1550 is concentrated. The input signal frequencies are from $500 \mathrm{~Hz}$ to $2 \mathrm{kHz}$ [105]. Through the way of phase generated carrier,signal information are extracted. These types are used for monitoring in borders . Micro-strain sensor taperd with a single mode fiber base photonic crystal fiber - single mode fiber structure is used . Fabrication process and sensing done by Intermittent cooling method for increased sensitivity with value $5.46 \mathrm{pm} / \mu \varepsilon$ [106].This provides more fessibility.

\section{S. DNA Hybridization}

Sensing DNA is difficult task because of its minimized structure. Such hybridization performed and viewed. In-line Michelson interferometer introduced with combination of tuneable mode coupler and opto fluidic channel. Guided light changes refractive index in optic fluid channel. Through binding of methylated DNA and 5-methylcytosine (5-mC) monoclonal antibody are identified. This work shows detection 5nM [107].Transformation from ssDNA into dsDNA, performed here. Amplitude with wavelength, 6.82 RIU-1 94.59 nm/RIU [108]. This sensor classifies gold and analyte for performing Hybridization.

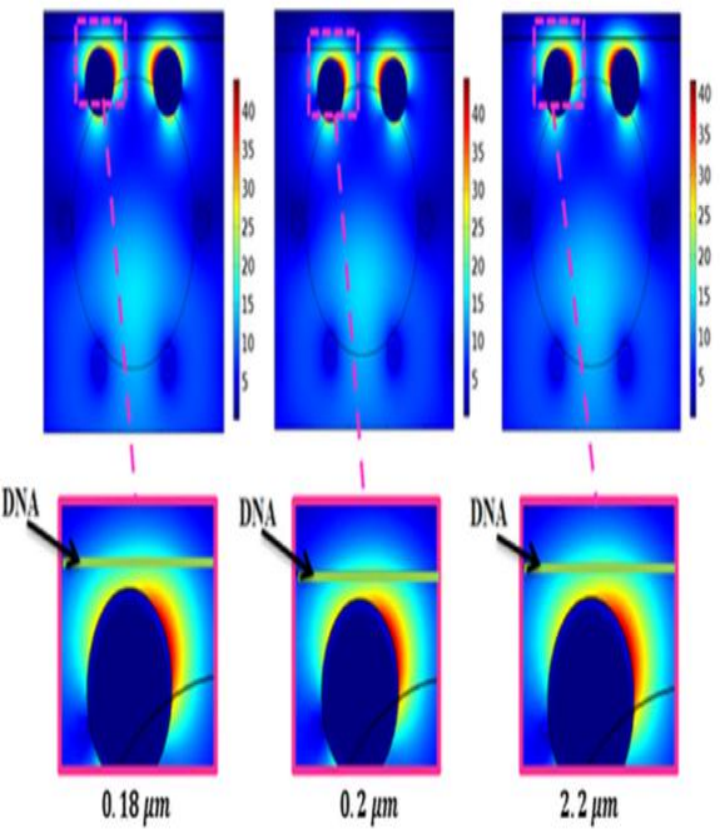

Fig 26. DNA Hybridization performance

\section{CONCLUSION}

Photonic crystal fibers are known from the year of 1996. As years pass on the necessity of Photonic crystal fiber are increased due its unique application in sensing field. In this paper we made a review on different sensors like physical, Curvature, Displacement, Electric and magnetic field, Refractive Index, Bio chemical, Biomedical which are used for sensing applications in Photonic crystal fiber. Different structure that are used in Photonic Crystal Fiber are analysed along with its sensitive range. Among all type of sensors, Sensing of Chemicals, Salinity in seawater and Blood component detection is comparatively a challenging task. As world grows upon civilization, new diseases also grow along. It became an essential to find the disease to avoid a pandemic situation. Testing Glucose in blood are performed level within a minutes. Similarly the testing of Disease should be done faster. To attain this we have to use different structures in future.

\section{ACKNOWLEDGMENT}

I would like to thank Revathi S, who guided me to write the article on Photonic crystal fiber, Also I thank Vellore Institute of Technology-Vellore for giving a chance to start my research work.

\section{REFERENCE}

1. Thomas F. Krauss, Richard M. De La Rue T.F. Krauss, R.M "Photonic crystals in the optical regime past, present and future" Progress in Quantum Electronics Volume 23 (1999) 5196.

2. Khushnub Anwar, Nowrin Nowsher, Basharat Nahar Islam "Analysis of Various PCF Structures Using Finite Element Method", Year 2017.

3. Moutusi De, Tarun Kumar Gangopadhyay, Vinod Kumar Singh "Prospects of Photonic Crystal Fiber as Physical Sensor: An Overview", Journal of sensors, Year 2019.

4. Min Shi, Shuguang Li,Hailiang Chen " $A$ high-sensitivity temperature sensor based on Sagnac interferometer employing photonic crysta fiber fully filled with ethanol" ,Journal on Applied Physics, Year 2018 ,Volume-124:94.

5. Changxu Li, Wenlong Yang, Xiaoyang Yu, Jianying Fan, Yanling Xiong,Yuqiang Yang, Linjun Li2" knotted shape core cladding optical fiber interferometer for Simultaneous Measurement of Displacement and Temperature", Year 2020.

6. Jin Wangb, Bo Liua,, Yongfeng Wua, Yaya Maoa, Lilong Zhaoa, Tingting Suna, Tong Nana "A novel fiber in-line Michelson interferometer based on end face packaging for temperature and refractive index measurement", Optik - International Journal for Light and Electron Optics 194 (2019) 163094.

7. Li Zhaoa, Yundong Zhanga, Yunhao Chena, Jinfang Wangb "Composite cavity fiber tip Fabry-Perot interferometer for high temperature Sensing” Optical Fiber Technology 50 (2019) page 3135.

8. Rami Zegadi \& Lahcène Ziet \& Ameur Zegadi "Design of High Sensitive Temperature Sensor Based on Two-Dimensional Photonic Crystal", Journal of Silicon, Year 2019.

9. Chao Du, Qi Wang, Yong Zhao "Electrically Tunable Long Period Gratings Temperature Sensor Based on Liquid Crystal Infiltrated Photonic Crystal " Journal of Fibers Sensors and Actuators, Year 2018.

10. "Fiber temperature and humidity sensor based on photonic crystal fiber coated with graphene oxide" Optics Communications 467 (2020) 125707.

11. Peter Tatar, Daniel Kacik, Norbert Tarjanyi "Fluorescein filled photonic crystal fiber sensor for simultaneous ultraviolet light and temperature monitoring", Optical Fiber Technology Volume, 30 Year 2016, Page 811.

Published By:

Blue Eyes Intelligence Engineering

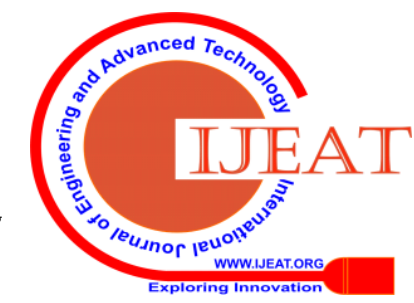




\section{Photonic Crystal Fiber for Sensing Application}

12. N. Ayyanar, R. Vasantha Jayakantha Raja, D. Vigneswaran, B. Lakshmi,M. Sumathi, K. Porsezian "Highly efficient compact temperature sensor using liquid infiltrated asymmetric dual elliptical core photonic crystal fiber" , Optical Materials Volume 64 (2017) $574 \mathrm{e} 582$.

13. Hai Liu, Haoran Wang, Cancan Chen, Wen Zhang, Sheng Zhang, Qing Wang, Yi Ding "Highly sensitive and temperature-compensated fiber bending sensing based on directional resonance coupling in (2019), Pages 164-171.

14. Chao Dua, Qi Wanga, Yong Zhao, Jin Li a "Highly sensitive temperature sensor based on an isopropanol-filled photonic crystal fiber long period grating", Optical Fiber Technology, Volume 34 (2017) ,Page 12-15.

15. Bikash Kumar paul,Md. Shadidul Islam, Kawsar ahmed and Sayed Asaduzzaman "Alcohol Sensing Over $O+E+S+C+L+U$ Transmission Band", Journal of Photonic sensors Vol. 7, No. 2, 2017: 123-130

16. Fengyan Wanga, Zuogang Zhub, Min Xuea, Fei Xuea, Qiuhong Wanga, Zihui Menga,Wei Lua, Wei Chena, Fenglian Qia, Zequn Yanaa "Cellulose photonic crystal film sensor for alcohols", Journa of Sensors and Actuators B 220 (2015) page 222-226.

17. Bikash Kumar Paul, Kawsar Ahmed, Vigneswaran Dhasarathan, Truong Khang Nguyen "Oligoporous-core Quasi cladding photonic crystal fiber based micro-sensor for alcohol detection", Physica B: Physics of Condensed Matter, Year 2019

18. Jakeya Sultana, MD. Saiful Islam, Kawsar Ahmed, Alex Dinovitser, "Terahertz detection of alcohol using a photonic crystal fiber sensor",

19. Md. Faizul Huq Arif, Md. Jaminul Haque Biddut "A new structure of photonic crystal fiber with high sensitivity, high nonlinearity, high birefringence and low confinement loss for liquid analyte sensing applications" Sensing and Bio-Sensing Research, Volume 12 (2017) pages 8-14.

20. Kawsar Ahmed Monir Morshed Sayed Asaduzzaman Md. Faizul Huq Arif "Optimization and enhancement of liquid analyte sensing

21. A. Dixita, S. Tiwaria, U. Ramania, P.C. Pandeya, Department "Refractive index sensor based on evanescent field effects in hollow core PCF for detection of analytes over extended $E+S+C+L+U$ communication ,bands", Optics and Laser Technology, Volume 121 (2020) 105779.

22. H. Ademgil " Simultaneous Sensing of Dual Analyte with Photonic Crystal Fiber Based Liquid Sensor", Balkan journal of electrical \& computer engineering, Volume. 7, No. 4, October 2019.

23. G. Dyankov, T.A. Eftimov, N. Malinowski, E. Belina, H. Kisov, P. Mikulic, W.J. Bock "A highly efficient biosensor based on MAPLE deposited hemoglobin on LPGs around phase matching turning point" Optics and Laser Technology Volume 123 (2020) 105907.

24. Ahmad Kamal Ahmad, "Bio-sensing simulations using HC-PCF filled with different bio-liquids" year 2020.

25. Md. Bellal Hossain·,Etu Podder, "Design and investigation of PCF-based blood components sensor in terahertz regime”, Applied Physics A Volume,125:861, Year 2019.

26. Veerpal Kaur, Surinder Singh "Design approach of solid-core photonic crystal fiber sensor with sensing ring for blood component detection", Journal of Nanophotonics ,Year 2019.

27. Kawsar Ahmed, Bikash Kumar Paula, B. Vasudevand, Ahmed Nabih Zaki Rashed, Maheswar. Amirig, P. Yupaping "Design of D-shaped Physics Volume 12 (2019) ,Pages 2021-2025 cell sensing application.

28. C.S. Boopathi, K. Vinoth Kumar, S. Sheebaranic, K. Selvakumar, Ahmed Nabih Zaki Rashedd,P. Yupapine, "Design of human blood sensor using symmetric dual core photonic crystal Fiber." Results in Physics Vol. 11 (2018) ,Pages 964-965.

29. Poonam Sharma, PreetaSharan ,"Design of photonic crystal based ring resonator for detection of different blood constituents", Optics Communications, Volume 348(2015), Pages 19-23.

30. A. Esther Lidiyaa, R. Vasantha Jayakantha Rajaa, Van Dai Pham, Quang Minh Ngob D. Vigneswarand "Detecting hemoglobin content blood glucose using surface plasmon resonance in D-shaped photonic crystal fiber", Optical Fiber Technology, Volume 50 (2019) pages 132-138.

31. N. Ayyanar,Ahmed E. Khalil, Mohamed Farhat O. Hameed G. Thavasi Raja ,Salah S. A. Obayya, "Enhanced sensitivity of photonic crystal fibers", Optical Fiber Technology Volume 47 Journal of Applied Optics, Year 2018. performance based on square-cored octagonal photonic crystal fiber", Journal of Optics, Year 2016. elliptical core photonic crystal fiber for blood plasma", Results in

hemoglobin sensor using dual-core photonic crystal fiber", Optical and Quantum Electronics (2018) Volume 50:453.

32. Crystal.P. Swain, G. Palai "Estimation of human-hemoglobin using honeycomb structure:An application of photonic crystal" , Optik,Volume 127 (2016) Pages 3333-3336.

33. Haoran Wanga, Wen Zhanga, Cancan Chena, Shoufeng Tanga, Hai Liua, "A new methane sensor based on compound film-coated photonic crystal fiber and Sagnac interferometer with higher sensitivity", Results in Physics, Volume 15 (2019) 102817.

34. Md. Biplob Hossain, Tamanna, Lway F. Abdulrazak,Md. Masud Rana, and Md. Rabiul Islama "A Numerical Approach to Design the Kretschmann Configuration Based Refractive Index Graphene-MoS2 Hybrid Layers With TiO2-SiO2 Nano for Formalin Detection", Photonic Sensors, Volume 10, No. 2, 2020: pages 134-146.

35. Md. Bellal Hossaina, Etu Poddera, Abdullah Al-Mamun Bulbula, Himadri Shekhar Mondala "Bane chemicals detection through photonic crystal fiber in $\mathrm{THz}$ regime", Optical Fiber Technology Volume 54 (2020) 102102.

36. Etu Podder, Md. Bellal Hossain, Rayhan Habib Jibon, Abdullah AlMamun Bulbul ,Himadri Shekhar Mondal ,"Chemical sensing through photonic crystal fiber: sulfuric acid Detection”, Optoelectron. 2019, Volume 12(4): Pages 372-381.

37. Sergey A. Pidenko , Natalia A. Burmistrova Pavel S. Pidenko , Andrey A. Shuvalov,Anastasiya A. Chibrova ,Yulia, Skibina, Irina Y. Goryacheva a, eControlled chemical modification of the internal surface of photonic, Optical Materials 60 (2016) 283e289.

38. Kawsar Ahmed, Bikash KumarPaul Sawrab Chowdhury, Md.ShadidulIslam, Shuvo Sen , Md. IbadulIslam, SayedAsaduzzaman , AliNewazBahar, Mohammad Badrul Alam Miah "A Dataset on photonic crystal fiber based chemical sensor crystal fibers for application as biosensitive elements", Data in Brief Volume 12(2017), Pages 227-233.

39. Boyao Li \& Meng Wu \& Xinyu Liu Guiyao Zhou \& Teng Wang \& Zicheng Sheng \& Zhiyun Hou \& Changming Xia , “ Design and Characterization of Bio-Chemical Sensor Based on Photonic Crysta Fiber with Fluorine-Doped Tin Oxides Film", Plasmonics (2019) Volume 14: Pages 197-203.

40. Sneha Sharma, Vijay Shanker Chaudhary, Dharmendra Kumar , “ Design of chemical sensor based on dual core photonic crystal fiber", Journal of Materials Today.

41. Shuvo Sen, Kawsar Ahmed, "Design of terahertz spectroscopy based optical sensor for chemical Detection”, Springer Nature,2019.

42. Malavikaa,. Prabub, "Design optimization of a highly sensitive spiral photonic crystal fiber for liquid and chemical sensing applications", Volume of Optical Fiber Technology Volume 51 (2019) Pages 3640.

43. Hai Liu, Haoran Wang, Cancan Chen,Wen Zhang, Bingbing Bai,Cong Chen, Yanzeng Zhang, Qiyuan Shao "High sensitive methane sensor based on twin-core photonic crystal fiber with compound film-coated side-holes", Optical and Quantum Electronics (2020) Pages 52:81.

44. Hai Liu, Haoran Wang, Wen Zhang, Cancan Chen, Qing Wang,Yi Ding, and Shoufeng Tang High Sensitive "Methane Sensor With Temperature, Compensation Based on Selectively Liquid-Infiltrated Photonic Crystal Fibers" Photonic Sensors Volume. 9, No. 3, 2019, Pages 213-222.

45. Hongze Gao , Guochen Wang, Wei Gao , She Li "A chiral photonic crystal fiber sensing coil for decreasing the polarization", Optics Communications, Volume 469 (2020) 125755.

46. Wen Wei Chen, Chun Liu Zhao, Zhao Kun Wang, Bang Ning Mao, "Curvature sensor based on SMF taper in fiber loop ring down system, Optical Fiber Technology 52 (2019) 101977.

47. Syed Mohammad Atiqullaha, Apu Palita, Mohammad Istiaque Rejaa, Jobaida Akhtara, Saleha Fatemab, Rubaya Absarc "Detection of harmful food additives using highly sensitive photonic crystal Fiber", Sensing and Bio-Sensing Research, Volume 23 (2019) 100275.

48. Md. Ibadul Islam, Kawsar Ahmed,Shuvo Sen, Sawrab Chowdhury,Bikash Kumar Pauk, Md. Shadidul Islam, Mohammad Badrul Alam Miah, Sayed, "Design and Optimization of Photonic Crystal Fiber Based Sensor for Gas Condensate and Air Pollution Monitoring", Photonic Sensors, Volume 7, No. 3, Year 2017: Pages 234-245.

Published By:

Blue Eyes Intelligence Engineering

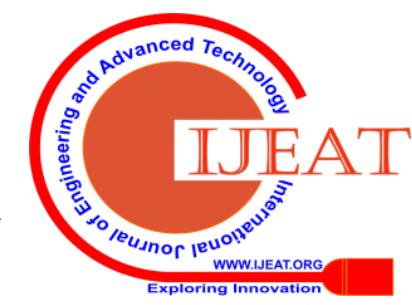


49. Afrooz Afsari , Mohammad Javadian Sarraf "Design of a hydrogen sulfide gas sensor based on a photonic crystal cavity using graphene" , Superlattices and Microstructures Volume 138 ,Year 2020, 106362.

50. Afrooz Afsari, Mohammad Javadian Sarraf "Design of a hydrogen sulfide gas sensor based on a photonic crystal cavity usingGraphene", Superlattices and Microstructures, Year 2019.

51. Hai Liu, Wen Zhang, Haoran Wang, Cancan Chena, Shoufeng Tang "Design of methane sensor based on slow light effect in hollow core

52. Jin Penga, Jie Liaoa, Xiaozhan Yanga, Wenlin Fenga, "Fiber-optic dual Fabry-Pérot interferometric carbon monoxide sensor with polyaniline /Co3O4/ graphene oxide sensing membrane” , Journal of Chemical Letters, Year 2019.

53. Md. Ibadul Islama, Bikash Kumar Paul, Kawsar Ahmed ,Md. Rabiul Hasan,Sawrab Chowdhurya, Md. Shadidul Islama, Shuvo Sena, Al Newaz Bahara, Sayed Asaduzzamana " Highly birefringent single mode spiral shape photonic crystal fiber based sensor for gas sensing applications" Sensing and Bio-Sensing Research ,Volume 14 (2017) pages. 30-38.

54. Xiaomei Shib, Zongshan Zhaoc, Zhanghua Hana, "Highly sensitive and selective gas sensing using the defect mode of a compact terahertz photonic crystal cavity", Sensors \& Actuators: B. Chemical Volume 274 Year 2018, Pages 188-193.

55. Ahmed Saber H. Rabeea, Mohamed Farhat O. Hameed Ahmed M. Heikal, S.S.A. Obayya "Highly sensitive photonic crystal fiber gas sensor", Optik - International Journal for Light and Electron Optics ,Volume 188 (2019) ,Pages 78-86.

56. X.C.Yang, Y.Lu, M.T.Wang, J.Q.Yao, " A photonic crystal fiber glucosesensor filled with silver nanowires", Optics Communications, Volume 359(2016)279-284.

57. S.Suganya ,P.Sangeetha, “ Design of Multi-Core Glucose Sensor Using Photonic Crystal Fiber", Asian Journal of Applied Science and Technology (AJAST), Volume 2, Issue 2, Pages 658-664, April-June 2018

58. Harikesavan Thenmozhia, Murugan Senthil Mani Rajana, Veluchamy Devikaa, Dhasarathan Vigneswaranb, Natesan Ayyanarc, "D-glucose sensor using photonic crystal fiber" , Journal of Optik, Volume 145 Year 2017, Pages 489-494.

59. Guowen An , Shuguang Li , Yinghong An , Haiyang Wang, Xuenan Zhang, "A Glucose sensor realized with photonic crystal fiber-based Sagnac interferometer", Optics Communications Volume 405 (2017), Pages. 143-146.

60. Qingshan Yanga, Guo Zhua, Lokendra Singh Yu Wanga, Ragin Singh Bingyuan Zhanga, Xia Zhanga, Santosh Kumara, "Highly sensitive and selective sensor probe using glucose oxidase gold nanoparticles/graphene oxide functionalized tapered optical fiber structure for detection of glucose", Optik - International Journal for Light and Electron Optics Volume 208 (2020) 164536.

61. S. Maheswaran, P.G. Kuppusamy, S.M. Ramesh, T.V.P. Sundararajand, P. Yupapine, "Refractive index sensor using dual core photonic crystal fiber - glucose detection applications", Results in Physics 11 (2018) pages 577-578.

62. Matthew Hartings, Kevin, Douglassa, Claire Neicea, Zeeshan Ahmeda, "Humidity responsive photonic sensor based on a carboxy methyl cellulose mechanical actuator", Sensors and Actuators B ,Volume 265 (2018) pages 335-338.

63. Diego Lopez-Torresa, Cesar Elosuaa, Joel Villatoroc,d, Joseba Zubiac,Manfred Rothhardte, Kay Schustere, Francisco J. Arreguia, "Photonic crystal fiber interferometer coated with a PAH/PAA nanolayer as humidity sensor", Journal of Sensors and Actuators B Year 2016.

64. Rui-jie Tonga, Yong Zhao, Mao-qing Chena, Yun Peng, "Relative humidity sensor based on small up-tapered photonic crystal fiber Mach-Zehnder interferometer", Sensors and Actuators A Volume 280 (2018), Pages 24-30.

65. Jiankun Penga, Weijia Wanga, Yapeng Qua, Tengpeng Suna, Dajuan Lva, Jixiang Daia,Minghong Yanga, "Thin films based onedimensional photonic crystal for humidity detection", Sensors and Actuators A,Volume 263 (2017),Pages 209-215.

66. Md. Faizul Huq Arif, Mohammad Mobarak Hossain, Nazrul Islam, Shah Mostaf Khaled "A nonlinear photonic crystal fiber for liquid sensing application with high birefringence and low confinement loss", Sensing and Bio-Sensing Research,Volume 22 (2019) 100252.

67. Revathi Senthil \& Anamika Soni \& Kushagra Bir \& Raghavee Senthil \& Prabu Krishnan ," Circular-Pattern Photonic Crystal Fiber for photonic crystal fiber".

Different Liquids with High Effective Area and Sensitivity", Plasmonics (2019) Volume 14, Pages 1783-1787.

68. Md. Jayed Bin Murshed Leon, Md. Ahasan Kabir "Design of a liquid sensing photonic crystal fiber with high sensitivity,bireferingence \& low confinement loss", Sensing and Bio-Sensing Research Volume 28 (2020) 100335

69. Ayad Zwayen Mohammed al-juboori , "Efficient optical fibre sensor for measuring Fe element in liquid", Optical Materials Volume 94 (2019), Pages 182-186.

70. Yan Luo, Xueqin Lei, Fuquan Shia, Baojin Peng, "A novel optical fiber magnetic field sensor based on Mach-Zehnder interferometer integrated with magnetic fluid", Optik - International Journal for Light and Electron Optics ,Volume174 (2018),Pages 252-258.

71. Sumaiya Akhtar Mitu \& Kawsar Ahmed \& Md. Nadim Hossain \& Bikash Kumar Paul \& Truong Khang Nguyen ,Vigneswaran Dhasarathan, "Design of Magnetic Fluid Sensor Using Elliptically Hole Assisted Photonic Crystal Fiber (PCF)", Journal of Superconductivity and Novel Magnetism.

72. Sumaiya Akhtar Mitua, Dipak Kumar Dey, Kawsar Ahmed, Bikash Kumar Paul,Yanhua Luod, R. Zakariae, Vigneswaran Dhasarathan, "Fe3O4 nanofluid injected photonic crystal fiber for magnetic field sensing Applications", Journal of Magnetism and Magnetic Materials, Volume 494 (2020) 165831.

73. Khadidja Saker, Touraya Bouchemat, Mahieddine Lahoubi, Mohamed Bouchemat, Shengli Pu, "Magnetic field sensor based on a magnetic fluid infiltrated photonic crystal L4 nanocavity and broadband W1 waveguide, Journal of Computational Electronics (2019) Volume 18: Pages 619-627

74. Yu Yinga, Nan Hua, Guang-yuan Sib, Ke Xua, Na Liuc, Ji-Zhong Zhaod "Magnetic field and temperature sensor based on D-shaped photonic crystal fiber", Optik - International Journal for Light and Electron Optics Volume 176 (2019), Pages 309-314.

75. Moutusi De, Vinod Kumar Singh, "Magnetic fluid infiltrated dua core photonic crystal fiber based highly sensitive magnetic field sensor", Optics and Laser Technology Volume 106 (2018): Pages 6168.

76. Qiang Liu Shu-Guang Li Xinyu Wang "Sensing characteristics of a MF-filled photonic crystal fiber Sagnac interferometer for magnetic field detecting", Sensors and Actuators B, Pages 2016.

77. Moutusi De, Akhilesh Kumar Pathak, Vinod Kumar Singh, " Single channel photonic crystal fiber based high sensitive petrol adulteration detection sensor ",Optik - International Journal for Light and Electron Optics , Volume 183 (2019), Pages 539-546.

78. Xuefeng Li, Han Zhang, Chunya Qian, Yanghui Ou, Runjie Shen, Hui Xiao "A new type of structure of optical fiber pressure sensor based on polarization modulation", Optics and Lasers in Engineering ,Volume 130 (2020) 106095

79. Zhen Yina, Rong Gaob, Youfu Gengc, Xiaoling Tand, Xuejin Li, “A reflective temperature-insensitive all-fiber polarization-mode interferometer and pressure sensing application based on PM-PCF" Optik , Volume 127 (2016) 9206-9211.

80. Yan Zhou, Yadong Denga, Lijuan Shea, Daru Chena, Zhangwei Yu, Zexuan Qiang, Wenshao Yanga, "Asymmetric double-core fiber for hydraulic pressure sensing” , Optical Fiber Technology Volume 48 (2019) Pages 194-198.

81. Vijay Shanker Chaudharya, Dharmendra Kumara,Rajan Mishra, Sneha Sharma, "Hybrid dual core photonic crystal fiber as hydrostatic pressure Sensor", Optik - International Journal for Light and Electron .

82. R. Rajasekar \& S. Robinson "Nano-Pressure and Temperature Sensor Based on Hexagonal Photonic Crystal Ring Resonator", Plasmonics (2019),Volume 14: Pages 3-15.

83. Yuefeng Q, Jin , Q Feng, Xin Zhang, Yanyan Liu, and Ying Han, “A Novel High Sensitivity Refractive Index Sensor Based on Multi-Core Micro/Nano Fiber", Photonic Sensors , Volume 9, No. 3, 2019: 197-204

84. Wan Zhang, Zhenggang Lian, Trevor Benson,Xin Wang, Shuqin Lou, "A refractive index sensor based on a D-shaped photonic crysta fiber with a nanoscale gold belt", Opt Quant Electron (2018) Volume $50: 29$

Published By:

Blue Eyes Intelligence Engineering

\& Sciences Publication 


\section{Photonic Crystal Fiber for Sensing Application}

85. Peng Gao, Yiping Gao, Meiyue , Songwei Liu, Yanan Zhang, "All-fiber Mach-Zehnder interferometer with dualwaist PCF structure for highly sensitive refractive index sensing", Applied Physics B (2019) Volume 125:107.

86. Matin Ashurov, Vladimir Gorelik, Kirill Napolski Sergey Klimonsky, "Anodic Alumina Photonic Crystals as Refractive Index Sensors for Controlling the Composition of Liquid Mixtures", Photonic Sensors Vol. 10, No. 2, 2020: 147-154.

87. Y.C. Tan Z.Q. Tou V. Mamidala K.K. Chow C.C.Continuous "Refractive Index Sensing based on Carbon-Nanotube-Deposited Photonic Crystal Fibers", Sensors and Actuators B,2014

88. Saawan K. Baga, Meher Wanb, Rajat K. Sinhac, Shailendra K. Varshney, "Design and characterization of surface relief grating on etchedmultimode optical fiber for refractive index sensing "Sensors and Actuators A 303 (2020) 111836

89. Chayma Mosbah, Ahlem Benmerkhi,Mohamed Bouchemat, Touraya Bouchemat" Design of refractive index sensing based on $2 \mathrm{D} \mathrm{PhC}$ air-slot width-modulated line-defect microcavity", Optical and Quantum Electronics (2019) 51:159.

90. Shivam Singh , Y. K. Prajapati, "Dual polarized ultrahigh sensitive gold/MoS2/graphene based D shaped PCF refractive index sensor in visible to nearIR region", Optical and Quantum Electronics (2020) 52:17

91. Shohag Khan, Kawsar Ahmed, Nadim Hossain, Bikash Kumar Paul,Truong Khang Nguyen, Vigneswaran Dhasarathan "Exploring Refractive index sensor using Gold coated D-shaped Photonic Crystal Fiber for biosensing applications", Journal of Optik,2019.

92. Haifeng , Xiaoyan , Youwang , Xinran, and Jianhang , "High Sensitive Refractive Index Sensor Based on Cladding Etched Photonic Crystal Fiber Mach-Zehnder Interferometer", Photonic Sensors ,Volume 9, No. 2, Year 2019: 126-134.

93. M.S. Aruna Gandhia, P. Ramesh Babub, K. Senthilnathanb, Qian Lia, " High sensitivity photonic crystal fiber-based refractive index microbiosensor, Optical Fiber Technology" Volume 46 (2018) ,Pages 88-94.

94. Yujun Wang, Shuguang Li, Jianshe Li, Zhen Zhang, Shuhuan Zhang, Junjun Wu "High-sensitivity photonic crystal fiber refractive index sensor based on directional coupler", Optical Fiber Technology 49 (2019) 16-21

95. Mahsa Vakili, Mina Noori, "Highly efficient elliptical microcavity refractive index sensor with single detection unit", Optical and Quantum Electronics (2019), 51:77.

96. Shivam Singh, Y. K. Prajapati, "Highly sensitive refractive index sensor based on D-shaped PCF with gold-graphene layers on the polished surface", Applied Physics A (2019) 125:437

97. A. K. Pathak \& S. Ghosh \& R. K. Gangwar \& B. M. A. Rahma, V. K. Singh "Metal Nanowire Assisted Hollow Core Fiber Sensor for an Efficient Detection of Small Refractive Index Change of Measurand Liquid", Plasmonics (2019) Volume 14:Pages 1823-1830.

98. Saeed Olyaee, Mahmood Seifouri, Rahim Karami, Ahmad Mohebzadeh-Bahabady "Designing a high sensitivity hexagonal nano-cavity photonic crystal resonator for the purpose of seawater salinity sensing”, Optical and Quantum Electronics (2019) 51:97.

99. Md. Aslam Mollaha, Md. Yousufalia, Md. Rifat Bin Asif Faysala, Md. Rabiul Hasanb, Md. Biplob Hossainc, I.S. Amirid, "Highly sensitive photonic crystal fiber salinity sensor based on Sagnac Interferometer", Results in Physics, Volume 16 (2020) 103022

100. D. Vigneswarana, N. Ayyanar, Mohit Sharma, M. Sumathi, M.S. Mani Rajand, Porseziane "Salinity sensor using photonic crystal fiber, Sensors and Actuators A Volume 269 (2018) Pages 22-28.

101. Sayantika Chowdhury, Sneha Verma, Tarun Kumar Gangopadhyay,"A comparative study and experimental observations of optical fiber sagnac interferometric based strain sensor by using different fibers", Optical Fiber Technology, Volume 48 (2019) Pages 283-288.

102. Fan Yang, Z.K. Wang, D.N. Wang, "A highly sensitive optical fiber strain sensor based on cascaded multimode fiber and photonic crystal fiber", Optical Fiber Technology Volume 47 (2019) 102-106.

103. Shuo Zhanga, Sifan Denga, Tao Genga, Cuiting Suna, Huiwen Niua, Xiaoyang Lia, Zemin Wanga,Xiang Lia, Yiwei Maa, Wenlei Yanga, Chengguo Tongb, Libo Yuan "A miniature ultra-long period fiber grating for simultaneous measurement of axial strain and temperature", Optics and Laser Technology 126 (2020) 106121.

104. Xuefeng Li, Han Zhang, Chunya Qian, Yanghui Ou, Runjie Shen, Hui Xiao, "A new type of structure of optical fiber pressure sensor based
105. Chuanbiao Zhang, Tigang Ning, Jingjing Zheng, Jian Xu, Xuekai Gao, Heng Lin, Jing Li, Li Pei, "An optical fiber strain sensor by using of taper based TCF structure”, Optics and Laser Technology Volume 120 (2019) 105687.

106. Adel Abdallah, "Experimental study on an interferometric strain sensor based on hollow-core photonic bandgap fiber for intrusion detection", Optics Communications Volume 428 (2018) Pages 35-40.

107. Ronghua Fan, Lianqin Lia, Yueyang Zhuo, Xiang Lva, Zhenjun Rena, Jianguo Shena, Baojin Penga,"Practical research on photonic crystal fiber micro-strain sensor, Optical Fiber Technology" Volume Volume 52 (2019) 101959.

108. Ran Gao , Dan-FengLu, JinCheng, YiJiang, LanJiang, Jian-DongXu , Zhi-MeiQi “ Fiber optofluidic biosensor for the label - free detection of DNA hybridization and methylation base donaninlinetunable mode coupler, Biosensors and Bioelectronics ,Volume 86(2016)321-329.

109. Mohammad Y, Azab, Mohamed Farhat, Hameed, Abed M. Nasr, S. S. A. Obayya," Label free detection for DNA hybridization using surface plasmon photonic crystal fiber biosensor" , Journal of Opt Quant Electron, Year 2018, 50:68ss.

\section{AUTHORS PROFILE}

R. Hemalatha, Research Scholar undergoing research in Optical Communication,VIT , Vellore, India.

Dr. S. Revathi Ph.D. in optical communication, M. Tech in Communication Engineering. VIT, Vellore, India. 